NRCPS-HE-05-77

\title{
Non-Abelian Tensor Gauge Fields
}

\author{
Generalization of Yang-Mills Theory
}

\author{
George Savvidy \\ Demokritos National Research Center \\ Institute of Nuclear Physics \\ Ag. Paraskevi, GR-15310 Athens,Greece \\ E-mail: savvidy@inp.demokritos.gr
}

\begin{abstract}
We suggest an extension of the gauge principle which includes tensor gauge fields. The extended non-Abelian gauge transformations of the tensor gauge fields form a new large group. On this group one can define field strength tensors, which are transforming homogeneously with respect to the extended gauge transformations. The invariant Lagrangian is quadratic in the field strength tensors and describes interaction of tensor gauge fields of arbitrary large integer spin $1,2, \ldots$. It does not contain higher derivatives of the tensor gauge fields, and all interactions take place through three- and four-particle exchanges with dimensionless coupling constant. In this extension of the Yang-Mills theory the vector gauge boson becomes a member of a bigger family of tensor gauge bosons.

We shall present a second invariant Lagrangian which can be constructed in terms of the above field strength tensors. The total Lagrangian is a sum of the two Lagrangians and exhibits enhanced local gauge invariance with double number of gauge parameters. This allows to eliminate all negative norm states of the nonsymmetric second-rank tensor gauge field, which describes therefore three physical polarizations: two symmetric polarizations of helicity-two massless charged tensor gauge bosons and antisymmetric polarization of helicity-zero charged B field.
\end{abstract}




\section{$1 \quad$ Introduction}

It is well understood, that the concept of local gauge invariance allows to define the nonAbelian gauge fields, to derive their dynamical field equations and to develop a universal point of view on matter interactions as resulting from the exchange of gauge quanta of different forms [1,2]. The fundamental forces - electromagnetic, weak and strong interactions - can successfully be described by the non-Abelian Yang-Mills fields. The vector-like gauge particles - the photon, $W^{ \pm}, Z$ and gluons - mediate interaction between smallest constituents of matter - leptons and quarks $[3,4,5,6,7,8,9,10,11,12,13,14$, $15,16,17,18,19,20,21,22]$.

The non-Abelian local gauge invariance, which was formulated by Yang and Mills in [1], requires that all interactions must be invariant under independent rotations of internal charges at all space-time points ${ }^{1}$. The gauge principle allows very little arbitrariness: the interaction of matter fields, which carry non-commuting internal charges, and the nonlinear self-interaction of gauge bosons are essentially fixed by the requirement of local gauge invariance, very similar to the self-interaction of gravitons in general relativity.

It is therefore appealing to extend the gauge principle, which was elevated by Yang and Mills to a powerful constructive principle, so that it will define the interaction of matter fields which carry not only non-commutative internal charges, but also arbitrary large spins. It seems that this will naturally lead to a theory in which fundamental forces will be mediated by integer-spin gauge quanta and that the Yang-Mills vector gauge boson will become a member of a bigger family of tensor gauge bosons.

In the previous paper [23] we extended the gauge principle so that it enlarges the original algebra of the Abelian local gauge transformations found in [60, 61, 62] to a nonAbelian case. The extended non-Abelian gauge transformations of the tensor gauge fields form a new large group. On this large group one can define field strength tensors, which are transforming homogeneously with respect to the extended gauge transformations. The invariant Lagrangian is quadratic in the field strength tensors and describes interaction of tensor gauge fields of arbitrary large integer spin $1,2, \ldots$

The purpose of the present paper is to present a second invariant Lagrangian which can be constructed in terms of the above field strength tensors. The total Lagrangian is a sum of the two Lagrangians and exhibits enhanced local gauge invariance with double number of gauge parameters. This allows to eliminate all negative norm states of the nonsymmetric second rank tensor gauge field $A_{\mu \lambda} \neq A_{\lambda \mu}$, which describes therefore three physical polarizations: two symmetric polarizations of helicity-two massless charged tensor gauge bosons and antisymmetric polarization of helicity-zero charged B field.

The early investigation of higher-spin representations of the Poincaré algebra and of the corresponding field equations is due to Majorana, Dirac and Wigner [30, 31, 33]. The theory of massive particles of higher spin was further developed by Fierz and Pauli [32] and Rarita and Schwinger [34]. The Lagrangian and S-matrix formulations of free field theory of massive and massless fields with higher spin have been completely constructed in $[35,36,37,38,39,40,41,42,43,44]$. The problem of introducing interaction appears to be much more complex $[45,46,47,48,49,50,56,57]$ and met enormous difficulties for spin fields higher than two $[51,52,53,54,55]$. The first positive result in this direction was the light-front construction of the cubic interaction term for the massless field of helicity $\pm \lambda$ in $[58,59]$.

\footnotetext{
${ }^{1}$ The early formulation of Abelian gauge invariance of QED was in [24, 25, 26, 27, 28] (see also [29]).
} 
In our approach the gauge fields are defined as rank- $(s+1)$ tensors

$$
A_{\mu \lambda_{1} \ldots \lambda_{s}}^{a}(x), \quad s=0,1,2, \ldots
$$

and are totally symmetric with respect to the indices $\lambda_{1} \ldots \lambda_{s}$. A priory the tensor fields have no symmetries with respect to the first index $\mu$. This is an essential departure from the previous considerations, in which the higher-rank tensors were totally symmetric $[32,36,41,43]$. The index $s$ runs from zero to infinity. The first member of this family of the tensor gauge bosons is the Yang-Mills vector boson $A_{\mu}^{a}$.

The extended non-Abelian gauge transformations of the tensor gauge fields are defined by the following equations [23]:

$$
\begin{aligned}
\delta A_{\mu}^{a} & =\left(\delta^{a b} \partial_{\mu}+g f^{a c b} A_{\mu}^{c}\right) \xi^{b} \\
\delta A_{\mu \nu}^{a} & =\left(\delta^{a b} \partial_{\mu}+g f^{a c b} A_{\mu}^{c}\right) \xi_{\nu}^{b}+g f^{a c b} A_{\mu \nu}^{c} \xi^{b}, \\
\delta A_{\mu \nu \lambda}^{a} & =\left(\delta^{a b} \partial_{\mu}+g f^{a c b} A_{\mu}^{c}\right) \xi_{\nu \lambda}^{b}+g f^{a c b}\left(A_{\mu \nu}^{c} \xi_{\lambda}^{b}+A_{\mu \lambda}^{c} \xi_{\nu}^{b}+A_{\mu \nu \lambda}^{c} \xi^{b}\right), \\
\ldots \ldots \ldots & . \ldots \ldots \ldots \ldots \ldots \ldots \ldots \ldots \ldots \ldots \ldots \ldots \ldots \ldots \ldots
\end{aligned}
$$

or in the general form by the formula

$$
\delta A_{\mu \lambda_{1} \ldots \lambda_{s}}^{a}=\left(\delta^{a b} \partial_{\mu}+g f^{a c b} A_{\mu}^{c}\right) \xi_{\lambda_{1} \lambda_{2} \ldots \lambda_{s}}^{b}+g f^{a c b} \sum_{i=1}^{s} \sum_{P^{\prime} s} A_{\mu \lambda_{1} \ldots \lambda_{i}}^{c} \xi_{\lambda_{i+1} \ldots \lambda_{s}}^{b},
$$

where the infinitesimal gauge parameters $\xi_{\lambda_{1} \ldots \lambda_{s}}^{b}$ are totally symmetric rank-s tensors. The summation $\sum_{P^{\prime} s}$ is over all permutations of two sets of indices $\lambda_{1} \ldots \lambda_{i}$ and $\lambda_{i+1} \ldots \lambda_{s}$ which correspond to nonequal terms. It is obvious that this transformation preserves the symmetry properties of the tensor gauge field $A_{\mu \lambda_{1} \ldots \lambda_{s}}^{a}$. Indeed, the first term in the r.h.s. is a covariant derivative of the totally symmetric rank-s tensor $\nabla_{\mu}^{a b} \xi_{\lambda_{1} \lambda_{2} \ldots \lambda_{s}}^{b}$ and every term $\sum_{P^{\prime} s} A_{\mu \lambda_{1} \ldots \lambda_{i}}^{c} \xi_{\lambda_{i+1} \ldots \lambda_{s}}^{b}$ in the second sum is totally symmetric with respect to the indices $\lambda_{1} \lambda_{2} \ldots \lambda_{s}$ by construction. The matrix form of the transformation is

$$
\delta_{\xi} A_{\mu \lambda_{1} \ldots \lambda_{s}}=\partial_{\mu} \xi_{\lambda_{1} \lambda_{2} \ldots \lambda_{s}}-i g\left[A_{\mu}, \xi_{\lambda_{1} \lambda_{2} \ldots \lambda_{s}}\right]-i g \sum_{i=1}^{s} \sum_{P^{\prime} s}\left[A_{\mu \lambda_{1} \ldots \lambda_{i}}, \xi_{\lambda_{i+1} \ldots \lambda_{s}}\right]
$$

where the tensor gauge fields are $A_{\mu \lambda_{1} \ldots \lambda_{s}}^{a b}=\left(L_{c}\right)^{a b} A_{\mu \lambda_{1} \ldots \lambda_{s}}^{c}=i f^{a c b} A_{\mu \lambda_{1} \ldots \lambda_{s}}^{c}$, and $L^{a}$ are the generators of the semisimple Lie group $\mathrm{G}$ in the adjoint representation.

These extended gauge transformations generate a closed algebraic structure. To see that, one should compute the commutator of two extended gauge transformations $\delta_{\eta}$ and $\delta_{\xi}$ of parameters $\eta$ and $\xi$. The commutator of two transformations can be expressed in the form [23]

$$
\left[\delta_{\eta}, \delta_{\xi}\right] A_{\mu \lambda_{1} \lambda_{2} \ldots \lambda_{s}}=-i g \delta_{\zeta} A_{\mu \lambda_{1} \lambda_{2} \ldots \lambda_{s}}
$$

and is again an extended gauge transformation with the gauge parameters $\{\zeta\}$ which are given by the matrix commutators

$$
\begin{aligned}
\zeta & =[\eta, \xi] \\
\zeta_{\lambda_{1}} & =\left[\eta, \xi_{\lambda_{1}}\right]+\left[\eta_{\lambda_{1}}, \xi\right] \\
\zeta_{\nu \lambda} & =\left[\eta, \xi_{\nu \lambda}\right]+\left[\eta_{\nu}, \xi_{\lambda}\right]+\left[\eta_{\lambda}, \xi_{\nu}\right]+\left[\eta_{\nu \lambda}, \xi\right]
\end{aligned}
$$


The generalized field strengths are defined as [23]

$$
\begin{aligned}
G_{\mu \nu}^{a} & =\partial_{\mu} A_{\nu}^{a}-\partial_{\nu} A_{\mu}^{a}+g f^{a b c} A_{\mu}^{b} A_{\nu}^{c}, \\
G_{\mu \nu, \lambda}^{a} & =\partial_{\mu} A_{\nu \lambda}^{a}-\partial_{\nu} A_{\mu \lambda}^{a}+g f^{a b c}\left(A_{\mu}^{b} A_{\nu \lambda}^{c}+A_{\mu \lambda}^{b} A_{\nu}^{c}\right), \\
G_{\mu \nu, \lambda \rho}^{a} & =\partial_{\mu} A_{\nu \lambda \rho}^{a}-\partial_{\nu} A_{\mu \lambda \rho}^{a}+g f^{a b c}\left(A_{\mu}^{b} A_{\nu \lambda \rho}^{c}+A_{\mu \lambda}^{b} A_{\nu \rho}^{c}+A_{\mu \rho}^{b} A_{\nu \lambda}^{c}+A_{\mu \lambda \rho}^{b} A_{\nu}^{c}\right),
\end{aligned}
$$

and transform homogeneously with respect to the extended gauge transformations (1). The field strength tensors are antisymmetric in their first two indices and are totaly symmetric with respect to the rest of the indices. By induction the entire construction can be generalized to include tensor fields of any rank s, and the corresponding field strength we shall define by the following expression:

$$
G_{\mu \nu, \lambda_{1} \ldots \lambda_{s}}^{a}=\partial_{\mu} A_{\nu \lambda_{1} \ldots \lambda_{s}}^{a}-\partial_{\nu} A_{\mu \lambda_{1} \ldots \lambda_{s}}^{a}+g f^{a b c} \sum_{i=0}^{s} \sum_{P^{\prime} s} A_{\mu \lambda_{1} \ldots \lambda_{i}}^{b} A_{\nu \lambda_{i+1} \ldots \lambda_{s}}^{c},
$$

where the sum $\sum_{P^{\prime} s}$ runs over all permutations of two sets of indices $\lambda_{1} \ldots \lambda_{i}$ and $\lambda_{i+1} \ldots \lambda_{s}$ which correspond to nonequal terms. All permutations of indices within two sets $\lambda_{1} \ldots \lambda_{i}$ and $\lambda_{i+1} \ldots \lambda_{s}$ correspond to equal terms, because gauge fields are totally symmetric with respect to $\lambda_{1} \ldots \lambda_{i}$ and $\lambda_{i+1} \ldots \lambda_{s}$. Therefore there are

$$
\frac{s !}{i !(s-i) !}
$$

nonequal terms in the sum $\sum_{P^{\prime} s}$. Thus in the sum $\sum_{P^{\prime} s}$ there is one term of the form $A_{\mu} A_{\nu \lambda_{1} \lambda_{2} \ldots \lambda_{s}}$, there are $s$ terms of the form $A_{\mu \lambda_{1}} A_{\nu \lambda_{2} \ldots \lambda_{s}}$ and $s(s-1) / 2$ terms of the form $A_{\mu \lambda_{1} \lambda_{2}} A_{\nu \lambda_{3} \ldots \lambda_{s}}$ and so on. In the above definition of the extended gauge field strength $G_{\mu \nu, \lambda_{1} \ldots \lambda_{s}}^{a}$, together with the classical Yang-Mills gauge boson $A_{\mu}^{a}$, there participate a set of higher-rank gauge fields $A_{\mu \lambda_{1}}^{a}, A_{\mu \lambda_{1}, \lambda_{2}}^{a}, \ldots, A_{\mu \lambda_{1} \ldots \lambda_{s}}^{a}$ up to the rank $s+1$. By construction the field strength (7) is antisymmetric with respect to its first two indices $G_{\mu \nu, \lambda_{1} \ldots \lambda_{s}}^{a}=-G_{\nu \mu, \lambda_{1} \ldots \lambda_{s}}^{a}$ and is totally symmetric with respect to the rest of the indices $G_{\mu \nu, . . \lambda_{i} \ldots \lambda_{j} . .}^{a}=G_{\mu \nu, \ldots \lambda_{j} \ldots \lambda_{i} . .}^{a}$, where $1 \leq i<j \leq s$.

The inhomogeneous extended gauge transformation (2) induces the homogeneous gauge transformation of the corresponding field strength (7) of the form [23]

$$
\begin{aligned}
\delta G_{\mu \nu}^{a} & =g f^{a b c} G_{\mu \nu}^{b} \xi^{c} \\
\delta G_{\mu \nu, \lambda}^{a} & =g f^{a b c}\left(G_{\mu \nu, \lambda}^{b} \xi^{c}+G_{\mu \nu}^{b} \xi_{\lambda}^{c}\right), \\
\delta G_{\mu \nu, \lambda \rho}^{a} & =g f^{a b c}\left(G_{\mu \nu, \lambda \rho}^{b} \xi^{c}+G_{\mu \nu, \lambda}^{b} \xi_{\rho}^{c}+G_{\mu \nu, \rho}^{b} \xi_{\lambda}^{c}+G_{\mu \nu}^{b} \xi_{\lambda \rho}^{c}\right)
\end{aligned}
$$

or in general

$$
\delta G_{\mu \nu, \lambda_{1} \ldots \lambda_{s}}^{a}=g f^{a b c} \sum_{i=0}^{s} \sum_{P^{\prime} s} G_{\mu \nu, \lambda_{1} \ldots \lambda_{i}}^{b} \xi_{\lambda_{i+1} \ldots \lambda_{s}}^{c} .
$$

The symmetry properties of the field strength $G_{\mu \nu, \lambda_{1} \ldots \lambda_{s}}^{a}$ remain invariant in the course of this transformation. The polygauge invariant Lagrangian now can be formulated in the form [23]

$$
\begin{aligned}
\mathcal{L}_{s+1} & =-\frac{1}{4} G_{\mu \nu, \lambda_{1} \ldots \lambda_{s}}^{a} G_{\mu \nu, \lambda_{1} \ldots \lambda_{s}}^{a}+\ldots \ldots \\
& =-\frac{1}{4} \sum_{i=0}^{2 s} a_{i}^{s} G_{\mu \nu, \lambda_{1} \ldots \lambda_{i}}^{a} G_{\mu \nu, \lambda_{i+1} \ldots \lambda_{2 s}}^{a} \sum_{p^{\prime} s} \eta^{\lambda_{i_{1}} \lambda_{i_{2}}} \ldots \ldots \eta^{\lambda_{i_{2 s-1}} \lambda_{i_{2 s}}}
\end{aligned}
$$


where the sum $\sum_{p}$ runs over all permutations of indices $\lambda_{1} \ldots \lambda_{2 s}$ which correspond to nonequal terms. For the low values of $s=0,1,2, \ldots$ the numerical coefficients

$$
a_{i}^{s}=\frac{s !}{i !(2 s-i) !}
$$

are: $a_{0}^{0}=1 ; \quad a_{1}^{1}=1, a_{0}^{1}=a_{2}^{1}=1 / 2 ; \quad a_{2}^{2}=1 / 2, a_{1}^{2}=a_{3}^{2}=1 / 3, a_{0}^{2}=a_{4}^{2}=1 / 12 ;$ and so on. In order to describe fixed rank- $(s+1)$ gauge field one should have at disposal all gauge fields up to the rank $2 s+1$. In order to make all tensor gauge fields dynamical one should add the corresponding kinetic terms. Thus the invariant Lagrangian describing dynamical tensor gauge bosons of all ranks has the form

$$
\mathcal{L}=\sum_{s=1}^{\infty} g_{s} \mathcal{L}_{s}
$$

where $\mathcal{L}_{1}$ is the Yang-Mills Lagrangian, $\mathcal{L}_{2}, \mathcal{L}_{3}, \ldots$ are new invariant forms quadratic in field strength tensors $(10)$ and $g_{s}$ are new coupling constants $\left(g_{1}=1\right)$.

The first three terms of the invariant Lagrangian have the following form [23]:

$$
\begin{aligned}
\mathcal{L}=\mathcal{L}_{1} & +g_{2} \mathcal{L}_{2}+g_{3} \mathcal{L}_{3}+\ldots=-\frac{1}{4} G_{\mu \nu}^{a} G_{\mu \nu}^{a}+g_{2}\left\{-\frac{1}{4} G_{\mu \nu, \lambda}^{a} G_{\mu \nu, \lambda}^{a}-\frac{1}{4} G_{\mu \nu}^{a} G_{\mu \nu, \lambda \lambda}^{a}\right\} \\
& +g_{3}\left\{-\frac{1}{4} G_{\mu \nu, \lambda \rho}^{a} G_{\mu \nu, \lambda \rho}^{a}-\frac{1}{8} G_{\mu \nu, \lambda \lambda}^{a} G_{\mu \nu, \rho \rho}^{a}-\frac{1}{2} G_{\mu \nu, \lambda}^{a} G_{\mu \nu, \lambda \rho \rho}^{a}-\frac{1}{8} G_{\mu \nu}^{a} G_{\mu \nu, \lambda \lambda \rho \rho}^{a}\right\}+. .,(12
\end{aligned}
$$

where the first term is the Yang-Mills Lagrangian and the second and the third ones describe the tensor gauge fields $A_{\mu \nu}^{a}, A_{\mu \nu \lambda}^{a}$ and so on. It is important that: i) the Lagrangian does not contain higher derivatives of tensor gauge fields ii) all interactions take place through the three- and four-particle exchanges with dimensionless coupling constant $g$ iii) the complete Lagrangian contains all higher-rank tensor gauge fields and should not be truncated iv) $g_{s}$ are new coupling constants.

\section{Enhanced Local Gauge Symmetry}

The Lagrangian (10), (11) and (12) is not the most general Lagrangian which can be constructed in terms of the above field strength tensors (6) and (7). We shall see that there exists a second invariant Lagrangian $\mathcal{L}_{2}^{\prime}$ which can be constructed for the second-rank nonsymmetric tensor gauge field $A_{\mu \lambda}^{a} \neq A_{\lambda \mu}^{a}$ and that the total Lagrangian is a sum of two Lagrangians $\mathcal{L}_{2}+\mathcal{L}_{2}^{\prime}$ and exhibits a new enhanced gauge symmetry (17) which allows to eliminate all negative norm polarizations of the nonsymmetric second-rank tensor gauge field $A_{\mu \lambda}$.

The invariance of the Lagrangian

$$
\mathcal{L}_{2}=-\frac{1}{4} G_{\mu \nu, \lambda}^{a} G_{\mu \nu, \lambda}^{a}-\frac{1}{4} G_{\mu \nu}^{a} G_{\mu \nu, \lambda \lambda}^{a}
$$

in (10), (11) and (12) was demonstrated in [23] by calculating its variation with respect to the gauge transformation (1) and (8), (9). Indeed, its variation is equal to zero

$$
\begin{aligned}
\delta \mathcal{L}_{2}= & -\frac{1}{2} G_{\mu \nu, \lambda}^{a} g f^{a b c}\left(G_{\mu \nu, \lambda}^{b} \xi^{c}+G_{\mu \nu}^{b} \xi_{\lambda}^{c}\right)-\frac{1}{4} g f^{a b c} G_{\mu \nu}^{b} \xi^{c} G_{\mu \nu, \lambda \lambda}^{a} \\
& -\frac{1}{4} G_{\mu \nu}^{a} g f^{a b c}\left(G_{\mu \nu, \lambda \lambda}^{b} \xi^{c}+2 G_{\mu \nu, \lambda}^{b} \xi_{\lambda}^{c}+G_{\mu \nu}^{b} \xi_{\lambda \lambda}^{c}\right)=0 .
\end{aligned}
$$


But the Lagrangian $\mathcal{L}_{2}$ is not a unique one, there exist additional Lorentz invariant quadratic forms which can be constructed by the corresponding field strength tensors. They are

$$
G_{\mu \nu, \lambda}^{a} G_{\mu \lambda, \nu}^{a}, \quad G_{\mu \nu, \nu}^{a} G_{\mu \lambda, \lambda}^{a}, \quad G_{\mu \nu}^{a} G_{\mu \lambda, \nu \lambda}^{a} .
$$

Calculating the variation of each of these terms with respect to the gauge transformation (1) and (8) one can get convinced that a particular linear combination

$$
\mathcal{L}_{2}^{\prime}=\frac{1}{4} G_{\mu \nu, \lambda}^{a} G_{\mu \lambda, \nu}^{a}+\frac{1}{4} G_{\mu \nu, \nu}^{a} G_{\mu \lambda, \lambda}^{a}+\frac{1}{2} G_{\mu \nu}^{a} G_{\mu \lambda, \nu \lambda}^{a}
$$

forms an invariant Lagrangian. Indeed, the variation of the Lagrangian $\mathcal{L}_{2}^{\prime}$ under the gauge transformation (8) is equal to zero

$$
\begin{aligned}
\delta \mathcal{L}_{2}^{\prime}= & +\frac{1}{4} G_{\mu \nu, \lambda}^{a} g f^{a b c}\left(G_{\mu \lambda, \nu}^{b} \xi^{c}+G_{\mu \lambda}^{b} \xi_{\nu}^{c}\right)+\frac{1}{4} g f^{a b c}\left(G_{\mu \nu, \lambda}^{b} \xi^{c}+G_{\mu \nu}^{b} \xi_{\lambda}^{c}\right) G_{\mu \lambda, \nu}^{a} \\
& +\frac{1}{2} G_{\mu \nu, \nu}^{a} g f^{a b c}\left(G_{\mu \lambda, \lambda}^{b} \xi^{c}+G_{\mu \lambda}^{b} \xi_{\lambda}^{c}\right) \\
& +\frac{1}{2} g f^{a b c} G_{\mu \nu}^{b} \xi^{c} G_{\mu \lambda, \nu \lambda}^{a} \\
& +\frac{1}{2} G_{\mu \nu}^{a} g f^{a b c}\left(G_{\mu \lambda, \nu \lambda}^{b} \xi^{c}+G_{\mu \lambda, \nu}^{b} \xi_{\lambda}^{c}+G_{\mu \lambda, \lambda}^{b} \xi_{\nu}^{c}+G_{\mu \lambda}^{b} \xi_{\nu \lambda}^{c}\right)=0 .
\end{aligned}
$$

As a result we have two invariant Lagrangians $\mathcal{L}_{2}$ and $\mathcal{L}_{2}^{\prime}$ and the general Lagrangian is a linear combination of these two invariant forms $g_{2} \mathcal{L}_{2}+g_{2}^{\prime} \mathcal{L}_{2}^{\prime}$, where $g_{2}$ and $g_{2}^{\prime}$ are arbitrary constants.

Our aim now is to demonstrate that if $g_{2}=g_{2}^{\prime}$ then we shall have enhanced local gauge invariance (17) of the Lagrangian $\mathcal{L}_{2}+\mathcal{L}_{2}^{\prime}$ with double number of gauge parameters. This allows to eliminate all negative norm states of the nonsymmetric second-rank tensor gauge field $A_{\mu \lambda}^{a} \neq A_{\lambda \mu}^{a}$, which describes therefore three physical modes: two symmetric polarizations of helicity-two massless charged tensor gauge bosons and antisymmetric polarization of helicity-zero charged B field.

Indeed let us consider the situation at the linearized level when the gauge coupling constant $\mathrm{g}$ is equal to zero. The free part of the $\mathcal{L}_{2}$ Lagrangian

$$
\mathcal{L}_{2}^{\text {free }}=\frac{1}{2} A_{\alpha \dot{\alpha}}^{a}\left(\eta_{\alpha \gamma} \eta_{\dot{\alpha} \dot{\gamma}} \partial^{2}-\eta_{\dot{\alpha} \dot{\gamma}} \partial_{\alpha} \partial_{\gamma}\right) A_{\gamma \dot{\gamma}}^{a}=\frac{1}{2} A_{\alpha \dot{\alpha}}^{a} H_{\alpha \dot{\alpha} \gamma \dot{\gamma}} A_{\gamma \dot{\gamma}}^{a},
$$

where the quadratic form in the momentum representation has the form

$$
H_{\alpha \dot{\alpha} \gamma \dot{\gamma}}(k)=\left(-k^{2} \eta_{\alpha \gamma}+k_{\alpha} k_{\gamma}\right) \eta_{\dot{\alpha} \dot{\gamma}}
$$

is obviously invariant with respect to the gauge transformation $\delta A_{\mu \lambda}^{a}=\partial_{\mu} \xi_{\lambda}^{a}$, but it is not invariant with respect to the alternative gauge transformations $\delta A_{\mu \lambda}^{a}=\partial_{\lambda} \eta_{\mu}^{a}$. This can be seen, for example, from the following relations in momentum representation:

$$
k_{\alpha} H_{\alpha \dot{\alpha} \gamma \hat{\gamma}}(k)=0, \quad k_{\dot{\alpha}} H_{\alpha \dot{\alpha} \gamma \dot{\gamma}}(k)=-\left(k^{2} \eta_{\alpha \gamma}-k_{\alpha} k_{\gamma}\right) k_{\dot{\gamma}} \neq 0 .
$$

Let us consider now the free part of the second Lagrangian

$$
\begin{array}{r}
\mathcal{L}_{2}^{\prime \text { free }}=\frac{1}{4} A_{\alpha \dot{\alpha}}^{a}\left(-\eta_{\alpha \dot{\gamma}} \eta_{\dot{\alpha} \gamma} \partial^{2}-\right. \\
\eta_{\alpha \dot{\alpha}} \eta_{\gamma \dot{\gamma}} \partial^{2}+\eta_{\alpha \dot{\gamma}} \partial_{\dot{\alpha}} \partial_{\gamma}+\eta_{\dot{\alpha} \gamma} \partial_{\alpha} \partial_{\dot{\gamma}}+\eta_{\alpha \dot{\alpha}} \partial_{\gamma} \partial_{\dot{\gamma}}+ \\
\left.+\eta_{\gamma \dot{\gamma}} \partial_{\alpha} \partial_{\dot{\alpha}}-2 \eta_{\alpha \gamma} \partial_{\dot{\alpha}} \partial_{\dot{\gamma}}\right) A_{\gamma \dot{\gamma}}^{a}=\frac{1}{2} A_{\alpha \dot{\alpha}}^{a} H_{\alpha \dot{\alpha} \gamma \dot{\gamma}}^{\prime} A_{\gamma \dot{\gamma}}^{a},
\end{array}
$$


where

$H_{\alpha \dot{\alpha} \gamma \dot{\gamma}}^{\prime}(k)=\frac{1}{2}\left(\eta_{\alpha \dot{\gamma}} \eta_{\dot{\alpha} \gamma}+\eta_{\alpha \dot{\alpha}} \eta_{\gamma \dot{\gamma}}\right) k^{2}-\frac{1}{2}\left(\eta_{\alpha \dot{\gamma}} k_{\dot{\alpha}} k_{\gamma}+\eta_{\dot{\alpha} \gamma} k_{\alpha} k_{\dot{\gamma}}+\eta_{\alpha \dot{\alpha}} k_{\gamma} k_{\dot{\gamma}}+\eta_{\gamma \dot{\gamma}} k_{\alpha} k_{\dot{\alpha}}-2 \eta_{\alpha \gamma} k_{\dot{\alpha}} k_{\dot{\gamma}}\right)$.

It is again invariant with respect to the gauge transformation $\delta A_{\mu \lambda}^{a}=\partial_{\mu} \xi_{\lambda}^{a}$, but it is not invariant with respect to the gauge transformations $\delta A_{\mu \lambda}^{a}=\partial_{\lambda} \eta_{\mu}^{a}$ as one can see from analogous relations

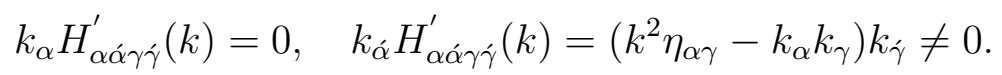

As it is obvious from (14) and (16), the total Lagrangian $\mathcal{L}_{2}^{\text {free }}+\mathcal{L}_{2}^{\prime}$ free now poses new enhanced invariance with respect to the larger, eight parameter, gauge transformations

$$
\delta A_{\mu \lambda}^{a}=\partial_{\mu} \xi_{\lambda}^{a}+\partial_{\lambda} \eta_{\mu}^{a}+\ldots
$$

where $\xi_{\lambda}^{a}$ and $\eta_{\mu}^{a}$ are eight arbitrary functions, because

$$
k_{\alpha}\left(H_{\alpha \dot{\alpha} \gamma \dot{\gamma}}+H_{\alpha \dot{\alpha} \gamma \dot{\gamma}}^{\prime}\right)=0, \quad k_{\dot{\alpha}}\left(H_{\alpha \dot{\alpha} \gamma \dot{\gamma}}+H_{\alpha \dot{\alpha} \gamma \dot{\gamma}}^{\prime}\right)=0 .
$$

Thus our free part of the Lagrangian is

$$
\begin{aligned}
\mathcal{L}_{2}^{\text {tot free }}= & -\frac{1}{2} \partial_{\mu} A_{\nu \lambda}^{a} \partial_{\mu} A_{\nu \lambda}^{a}+\frac{1}{2} \partial_{\mu} A_{\nu \lambda}^{a} \partial_{\nu} A_{\mu \lambda}^{a}+ \\
& +\frac{1}{4} \partial_{\mu} A_{\nu \lambda}^{a} \partial_{\mu} A_{\lambda \nu}^{a}-\frac{1}{4} \partial_{\mu} A_{\nu \lambda}^{a} \partial_{\lambda} A_{\mu \nu}^{a}-\frac{1}{4} \partial_{\nu} A_{\mu \lambda}^{a} \partial_{\mu} A_{\lambda \nu}^{a}+\frac{1}{4} \partial_{\nu} A_{\mu \lambda}^{a} \partial_{\lambda} A_{\mu \nu}^{a} \\
& +\frac{1}{4} \partial_{\mu} A_{\nu \nu}^{a} \partial_{\mu} A_{\lambda \lambda}^{a}-\frac{1}{2} \partial_{\mu} A_{\nu \nu}^{a} \partial_{\lambda} A_{\mu \lambda}^{a}+\frac{1}{4} \partial_{\nu} A_{\mu \nu}^{a} \partial_{\lambda} A_{\mu \lambda}^{a}
\end{aligned}
$$

or, in equivalent form, it is

$$
\begin{aligned}
\mathcal{L}_{2}^{\text {tot free }}=\frac{1}{2} A_{\alpha \dot{\alpha}}^{a}\left\{\left(\eta_{\alpha \gamma} \eta_{\dot{\alpha} \dot{\gamma}}\right.\right. & \left.-\frac{1}{2} \eta_{\alpha \dot{\gamma}} \eta_{\dot{\alpha} \gamma}-\frac{1}{2} \eta_{\alpha \dot{\alpha}} \eta_{\gamma \dot{\gamma}}\right) \partial^{2}-\eta_{\dot{\alpha} \dot{\gamma}} \partial_{\alpha} \partial_{\gamma}-\eta_{\alpha \gamma} \partial_{\dot{\alpha}} \partial_{\dot{\gamma}}+ \\
+ & \left.\frac{1}{2}\left(\eta_{\alpha \dot{\gamma}} \partial_{\dot{\alpha}} \partial_{\gamma}+\eta_{\dot{\alpha} \gamma} \partial_{\alpha} \partial_{\dot{\gamma}}+\eta_{\alpha \dot{\alpha}} \partial_{\gamma} \partial_{\dot{\gamma}}+\eta_{\gamma \dot{\gamma}} \partial_{\alpha} \partial_{\dot{\alpha}}\right)\right\} A_{\gamma \dot{\gamma}}^{a}
\end{aligned}
$$

and is invariant with respect to the larger gauge transformations $\delta A_{\mu \lambda}^{a}=\partial_{\mu} \xi_{\lambda}^{a}+\partial_{\lambda} \eta_{\mu}^{a}$, where $\xi_{\lambda}^{a}$ and $\eta_{\mu}^{a}$ are eight arbitrary functions. In momentum representation the quadratic form is

$$
\begin{aligned}
H_{\alpha \dot{\alpha} \gamma \dot{\gamma}}^{\text {tot }}(k)=\left(-\eta_{\alpha \gamma} \eta_{\dot{\alpha} \dot{\gamma}}\right. & \left.+\frac{1}{2} \eta_{\alpha \dot{\gamma}} \eta_{\dot{\alpha} \gamma}+\frac{1}{2} \eta_{\alpha \dot{\alpha}} \eta_{\gamma \dot{\gamma}}\right) k^{2}+\eta_{\alpha \gamma} k_{\dot{\alpha}} k_{\dot{\gamma}}+\eta_{\dot{\alpha} \dot{\gamma}} k_{\alpha} k_{\gamma} \\
& -\frac{1}{2}\left(\eta_{\alpha \dot{\gamma}} k_{\dot{\alpha}} k_{\gamma}+\eta_{\dot{\alpha} \gamma} k_{\alpha} k_{\dot{\gamma}}+\eta_{\alpha \dot{\alpha}} k_{\gamma} k_{\dot{\gamma}}+\eta_{\gamma \dot{\gamma}} k_{\alpha} k_{\dot{\alpha}}\right) .
\end{aligned}
$$

In summary, we have the following Lagrangian for the lower-rank tensor gauge fields:

$$
\begin{aligned}
\mathcal{L}=\mathcal{L}_{1}+g_{2}\left(\mathcal{L}_{2}+\mathcal{L}_{2}^{\prime}\right)= & -\frac{1}{4} G_{\mu \nu}^{a} G_{\mu \nu}^{a} \\
+ & g_{2}\left\{-\frac{1}{4} G_{\mu \nu, \lambda}^{a} G_{\mu \nu, \lambda}^{a}-\frac{1}{4} G_{\mu \nu}^{a} G_{\mu \nu, \lambda \lambda}^{a}+\right. \\
& \left.+\frac{1}{4} G_{\mu \nu, \lambda}^{a} G_{\mu \lambda, \nu}^{a}+\frac{1}{4} G_{\mu \nu, \nu}^{a} G_{\mu \lambda, \lambda}^{a}+\frac{1}{2} G_{\mu \nu}^{a} G_{\mu \lambda, \nu \lambda}^{a}\right\} .
\end{aligned}
$$


Let us consider the equations of motion which follow from this Lagrangian for the vector gauge field $A_{\nu}^{a}$

$$
\begin{gathered}
\nabla_{\mu}^{a b} G_{\mu \nu}^{b}+g_{2}\left\{\frac{1}{2} \nabla_{\mu}^{a b}\left(G_{\mu \nu, \lambda \lambda}^{b}+G_{\nu \lambda, \mu \lambda}^{b}+G_{\lambda \mu, \nu \lambda}^{b}\right)+g f^{a c b} A_{\mu \lambda}^{c} G_{\mu \nu, \lambda}^{b}\right. \\
-\frac{1}{2} g f^{a c b}\left(A_{\mu \lambda}^{c} G_{\mu \lambda, \nu}^{b}+A_{\mu \lambda}^{c} G_{\lambda \nu, \mu}^{b}+A_{\lambda \lambda}^{c} G_{\mu \nu, \mu}^{b}+A_{\mu \nu}^{c} G_{\mu \lambda, \lambda}^{b}-\right. \\
\left.\left.-A_{\mu \lambda \lambda}^{c} G_{\mu \nu}^{b}-A_{\mu \mu \lambda}^{c} G_{\nu \lambda}^{b}-A_{\mu \nu \lambda}^{c} G_{\lambda \mu}^{b}\right)\right\}=0
\end{gathered}
$$

and for the second-rank tensor gauge field $A_{\nu \lambda}^{a}$

$$
\begin{gathered}
\nabla_{\mu}^{a b} G_{\mu \nu, \lambda}^{b}-\frac{1}{2}\left(\nabla_{\mu}^{a b} G_{\mu \lambda, \nu}^{b}+\nabla_{\mu}^{a b} G_{\lambda \nu, \mu}^{b}+\nabla_{\lambda}^{a b} G_{\mu \nu, \mu}^{b}+\eta_{\nu \lambda} \nabla_{\mu}^{a b} G_{\mu \rho, \rho}^{b}\right) \\
+g f^{a c b} A_{\mu \lambda}^{c} G_{\mu \nu}^{b}-\frac{1}{2} g f^{a c b}\left(A_{\mu \nu}^{c} G_{\mu \lambda}^{b}+A_{\mu \mu}^{c} G_{\lambda \nu}^{b}+A_{\lambda \mu}^{c} G_{\mu \nu}^{b}+\eta_{\nu \lambda} A_{\mu \rho}^{c} G_{\mu \rho}^{b}\right)=0 .
\end{gathered}
$$

Representing these equations in the form

$$
\begin{gathered}
\partial_{\mu} F_{\mu \nu}^{a}+g_{2} \frac{1}{2} \partial_{\mu}\left(F_{\mu \nu, \lambda \lambda}^{a}+F_{\nu \lambda, \mu \lambda}^{a}+F_{\lambda \mu, \nu \lambda}^{a}\right)=j_{\nu}^{a}, \\
\partial_{\mu} F_{\mu \nu, \lambda}^{a}-\frac{1}{2}\left(\partial_{\mu} F_{\mu \lambda, \nu}^{a}+\partial_{\mu} F_{\lambda \nu, \mu}^{a}+\partial_{\lambda} F_{\mu \nu, \mu}^{a}+\eta_{\nu \lambda} \partial_{\mu} F_{\mu \rho, \rho}^{a}\right)=j_{\nu \lambda}^{a},
\end{gathered}
$$

where $F_{\mu \nu}^{a}=\partial_{\mu} A_{\nu}^{a}-\partial_{\nu} A_{\mu}^{a}, F_{\mu \nu, \lambda}^{a}=\partial_{\mu} A_{\nu \lambda}^{a}-\partial_{\nu} A_{\mu \lambda}^{a}, F_{\mu \nu, \lambda \rho}^{a}=\partial_{\mu} A_{\nu \lambda \rho}^{a}-\partial_{\nu} A_{\mu \lambda \rho}^{a}$, we can find the corresponding conserved currents

$$
\begin{aligned}
j_{\nu}^{a}= & -g f^{a b c} A_{\mu}^{b} G_{\mu \nu}^{c}-g f^{a b c} \partial_{\mu}\left(A_{\mu}^{b} A_{\nu}^{c}\right) \\
& -\frac{1}{2} g f^{a b c} A_{\mu}^{b}\left(G_{\mu \nu, \lambda \lambda}^{c}+G_{\nu \lambda, \mu \lambda}^{c}+G_{\lambda \mu, \nu \lambda}^{c}\right)-\frac{1}{2} \partial_{\mu}\left(I_{\mu \nu, \lambda \lambda}^{a}+I_{\nu \lambda, \mu \lambda}^{a}+I_{\lambda \mu, \nu \lambda}^{a}\right) \\
& -g f^{a b c} A_{\mu \lambda}^{b} G_{\mu \nu, \lambda}^{c}+\frac{1}{2} g f^{a b c}\left(A_{\mu \lambda}^{b} G_{\mu \lambda, \nu}^{c}+A_{\mu \lambda}^{b} G_{\lambda \nu, \mu}^{c}+A_{\lambda \lambda}^{b} G_{\mu \nu, \mu}^{c}+A_{\mu \nu}^{b} G_{\mu \lambda, \lambda}^{c}\right) \\
& -\frac{1}{2} g f^{a b c}\left(A_{\mu \lambda \lambda}^{b} G_{\mu \nu}^{c}+A_{\lambda \mu \lambda}^{b} G_{\nu \mu}^{c}+A_{\mu \lambda \nu}^{b} G_{\lambda \mu}^{c}\right),
\end{aligned}
$$

where $I_{\mu \nu, \lambda \rho}^{a}=g f^{a b c}\left(A_{\mu}^{b} A_{\nu \lambda \rho}^{c}+A_{\mu \lambda}^{b} A_{\nu \rho}^{c}+A_{\mu \rho}^{b} A_{\nu \lambda}^{c}+A_{\mu \lambda \rho}^{b} A_{\nu}^{c}\right)$ and

$$
\begin{aligned}
j_{\nu \lambda}^{a}= & -g f^{a b c} A_{\mu}^{b} G_{\mu \nu, \lambda}^{c}+\frac{1}{2} g f^{a b c}\left(A_{\mu}^{b} G_{\mu \lambda, \nu}^{c}+A_{\mu}^{b} G_{\lambda \nu, \mu}^{c}+A_{\lambda}^{b} G_{\mu \nu, \mu}^{c}+\eta_{\nu \lambda} A_{\mu}^{b} G_{\mu \rho, \rho}^{c}\right) \\
& -g f^{a b c} A_{\mu \lambda}^{b} G_{\mu \nu}^{c}+\frac{1}{2} g f^{a b c}\left(A_{\mu \nu}^{b} G_{\mu \lambda}^{c}+A_{\lambda \mu}^{b} G_{\mu \nu}^{c}+A_{\mu \mu}^{b} G_{\lambda \nu}^{c}+\eta_{\nu \lambda} A_{\mu \rho}^{b} G_{\mu \rho}^{c}\right) \\
& -g f^{a b c} \partial_{\mu}\left(A_{\mu}^{b} A_{\nu \lambda}^{c}+A_{\mu \lambda}^{b} A_{\nu}^{c}\right)+\frac{1}{2} g f^{a b c}\left[\partial_{\mu}\left(A_{\mu}^{b} A_{\lambda \nu}^{c}+A_{\mu \nu}^{b} A_{\lambda}^{c}\right)+\partial_{\mu}\left(A_{\lambda}^{b} A_{\nu \mu}^{c}+A_{\lambda \mu}^{b} A_{\nu}^{c}\right)\right. \\
& \left.+\partial_{\lambda}\left(A_{\mu}^{b} A_{\nu \mu}^{c}+A_{\mu \mu}^{b} A_{\nu}^{c}\right)+\eta_{\nu \lambda} \partial_{\mu}\left(A_{\mu}^{b} A_{\rho \rho}^{c}+A_{\mu \rho}^{b} A_{\rho}^{c}\right)\right] .
\end{aligned}
$$

Thus on mass shell

$$
\partial_{\nu} j_{\nu}^{a}=0, \quad \partial_{\nu} j_{\nu \lambda}^{a}=0, \quad \partial_{\lambda} j_{\nu \lambda}^{a}=0
$$

because, as we demonstrated above, the partial derivatives of the l.h.s.'s of the equations (25) are equal to zero (see equations (18)). 
At the linearized level, when the gauge coupling constant $\mathrm{g}$ is equal to zero, the equations of motion (25) for the second-rank tensor gauge fields will take the form

$$
\begin{aligned}
\partial^{2}\left(A_{\nu \lambda}^{a}-\frac{1}{2} A_{\lambda \nu}^{a}\right)-\partial_{\nu} \partial_{\mu}\left(A_{\mu \lambda}^{a}-\frac{1}{2} A_{\lambda \mu}^{a}\right) & -\partial_{\lambda} \partial_{\mu}\left(A_{\nu \mu}^{a}-\frac{1}{2} A_{\mu \nu}^{a}\right)+\partial_{\nu} \partial_{\lambda}\left(A_{\mu \mu}^{a}-\frac{1}{2} A_{\mu \mu}^{a}\right) \\
& +\frac{1}{2} \eta_{\nu \lambda}\left(\partial_{\mu} \partial_{\rho} A_{\mu \rho}^{a}-\partial^{2} A_{\mu \mu}^{a}\right)=0
\end{aligned}
$$

and, as we shall see below, describe the propagation of massless particles of spin 2 and spin 0. Indeed, it is easy to see that our equation reduces to the well known Fierz-PauliSchwinger-Chang-Singh-Hagen-Fronsdal equation for the symmetric part of the tensor gauge fields $A_{\nu \lambda}^{a}$

$$
\partial^{2} A_{\nu \lambda}-\partial_{\nu} \partial_{\mu} A_{\mu \lambda}-\partial_{\lambda} \partial_{\mu} A_{\mu \nu}+\partial_{\nu} \partial_{\lambda} A_{\mu \mu}+\eta_{\nu \lambda}\left(\partial_{\mu} \partial_{\rho} A_{\mu \rho}-\partial^{2} A_{\mu \mu}\right)=0
$$

which describes the propagation of massless boson with two physical polarizations, the $\lambda= \pm 2$ helicity states. For the antisymmetric part $A_{\nu \lambda}^{a}$ the equation reduces to the form

$$
\partial^{2} A_{\nu \lambda}-\partial_{\nu} \partial_{\mu} A_{\mu \lambda}+\partial_{\lambda} \partial_{\mu} A_{\mu \nu}=0
$$

and describes the propagation of massless tensor boson with one physical polarization, the $\lambda=0$ helicity state. Thus we have seen that the extended gauge symmetry is sufficient to exclude all negative norm polarizations from the spectrum of the second-rank nonsymmetric tensor gauge field $A_{\mu \lambda}$.

Using the gauge invariance (17) we can impose the Lorentz invariant supplementary conditions on the second-rank gauge fields $A_{\mu \lambda}: \partial_{\mu} A_{\mu \lambda}^{a}=a_{\lambda}, \quad \partial_{\lambda} A_{\mu \lambda}^{a}=b_{\mu}$, where $a_{\lambda}$ and $b_{\mu}$ are arbitrary functions, or one can suggest alternative supplementary conditions in which the quadratic form (19), (20), (21) is diagonal:

$$
\partial_{\mu} A_{\mu \lambda}^{a}-\frac{1}{2} \partial_{\lambda} A_{\mu \mu}^{a}=0, \quad \partial_{\lambda} A_{\mu \lambda}^{a}-\frac{1}{2} \partial_{\mu} A_{\lambda \lambda}^{a}=0
$$

In this gauge the equation has the form

$$
\partial^{2} A_{\nu \lambda}^{a}=0
$$

and in the momentum representation $A_{\mu \nu}(x)=e_{\mu \nu}(k) e^{i k x}$ from equation (31) it follows that $k^{2}=0$ and we have massless particles. In this gauge we shall get

$$
H_{\alpha \alpha \dot{\gamma} \gamma \hat{\gamma}}^{f i x}(k)=\left(\eta_{\alpha \gamma} \eta_{\dot{\alpha} \dot{\gamma}}-\frac{1}{2} \eta_{\alpha \dot{\alpha}} \eta_{\gamma \dot{\gamma}}\right)\left(-k^{2}\right)
$$

and can find the propagator $\Delta_{\gamma \dot{\gamma} \lambda \dot{\lambda}}(k)$ from the equation $H_{\alpha \alpha \dot{\gamma} \gamma \hat{\gamma}}^{f i x}(k) \Delta_{\gamma \dot{\gamma} \lambda \dot{\lambda}}(k)=\eta_{\alpha \lambda} \eta_{\dot{\alpha} \dot{\lambda}}$, thus

$$
\Delta_{\gamma \dot{\gamma} \lambda \dot{\lambda}}(k)=-\frac{\eta_{\gamma \lambda} \eta_{\dot{\gamma} \dot{\lambda}}-\frac{1}{2} \eta_{\gamma \dot{\gamma}} \eta_{\lambda \dot{\lambda}}}{k^{2}-i \varepsilon}
$$

The corresponding residue can be represented as a sum

$$
\eta_{\gamma \lambda} \eta_{\dot{\gamma} \hat{\lambda}}-\frac{1}{2} \eta_{\gamma \dot{\gamma}} \eta_{\lambda \dot{\lambda}}=+\frac{1}{2}\left(\eta_{\gamma \lambda} \eta_{\dot{\gamma} \dot{\lambda}}+\eta_{\gamma \dot{\lambda}} \eta_{\dot{\gamma} \lambda}-\eta_{\gamma \dot{\gamma}} \eta_{\lambda \dot{\lambda}}\right)+\frac{1}{2}\left(\eta_{\gamma \lambda} \eta_{\dot{\gamma} \dot{\lambda}}-\eta_{\gamma \dot{\lambda}} \eta_{\dot{\gamma} \lambda}\right) .
$$

The first term describes the $\lambda= \pm 2$ helicity states and is represented by the symmetric part of the polarization tensor $e_{\mu \lambda}$, the second term describes $\lambda=0$ helicity state and 
is represented by its antisymmetric part. When $k_{\mu}$ is aligned along the third axis, $k_{\mu}=$ $(k, 0,0, k)$, we have two independent polarizations of the helicity- 2 and helicity- 0 particles

$$
e_{\mu \lambda}^{1}=\frac{1}{\sqrt{2}}\left(\begin{array}{cc}
0,0, & 0,0 \\
0,1, & 0,0 \\
0,0,-1,0 \\
0,0, & 0,0
\end{array}\right), e_{\mu \lambda}^{2}=\frac{1}{\sqrt{2}}\left(\begin{array}{c}
0,0,0,0 \\
0,0,1,0 \\
0,1,0,0 \\
0,0,0,0
\end{array}\right), e_{\mu \lambda}^{A}=\frac{1}{\sqrt{2}}\left(\begin{array}{cc}
0, & 0,0,0 \\
0, & 0,1,0 \\
0, & -1,0,0 \\
0, & 0,0,0
\end{array}\right)
$$

with the property that $e_{\gamma \dot{\gamma}}^{1} e_{\lambda \dot{\lambda}}^{1}+e_{\gamma \dot{\gamma}}^{2} e_{\lambda \dot{\lambda}}^{2} \simeq \frac{1}{2}\left(\eta_{\gamma \lambda} \eta_{\dot{\gamma} \dot{\lambda}}+\eta_{\gamma \dot{\lambda}} \eta_{\dot{\gamma} \lambda}-\eta_{\gamma \dot{\gamma}} \eta_{\lambda \dot{\lambda}}\right)$ and $e_{\gamma \dot{\gamma}}^{A} e_{\lambda \dot{\lambda}}^{A} \simeq$ $\frac{1}{2}\left(\eta_{\gamma \lambda} \eta_{\dot{\gamma} \dot{\lambda}}-\eta_{\gamma \dot{\lambda}} \eta_{\dot{\gamma} \lambda}\right)$. The symbol $\simeq$ means that the equation holds up to longitudinal terms.

Thus the general second-rank tensor gauge field with 16 components $A_{\mu \lambda}$ describes in this theory three physical propagating massless polarizations.

\section{Interaction Vertices}

We are interested now to analyze the interaction properties of the tensor gauge bosons prescribed by the gauge principle. The interaction of the Yang-Mills vector bosons with the charged tensor gauge bosons is described by the Lagrangian $(22) \mathcal{L}_{2}+\mathcal{L}_{2}^{\prime}$. Let us first consider a three-particle interaction vertices - VTT. Explicitly three-linear terms of the Lagrangian $\mathcal{L}_{2}$ have the form:

$$
\begin{aligned}
\mathcal{L}_{2}^{c u b i c}= & -\frac{1}{2} g f^{a b c}\left(\partial_{\mu} A_{\nu \lambda}^{a}-\partial_{\nu} A_{\mu \lambda}^{a}\right)\left(A_{\mu}^{b} A_{\nu \lambda}^{c}+A_{\mu \lambda}^{b} A_{\nu}^{c}\right) \\
& -\frac{1}{4} g f^{a b c}\left(\partial_{\mu} A_{\nu}^{a}-\partial_{\nu} A_{\mu}^{a}\right) 2 A_{\mu \lambda}^{b} A_{\nu \lambda}^{c},
\end{aligned}
$$

and in addition to the standard Yang-Mills VVV three vector boson interaction vertex

$$
\mathcal{L}_{1}^{c u b i c}=-\frac{1}{2} g f^{a b c}\left(\partial_{\mu} A_{\nu}^{a}-\partial_{\nu} A_{\mu}^{a}\right) A_{\mu}^{b} A_{\nu}^{c}
$$

which in momentum representation has the form

$$
\mathcal{V}_{\alpha \beta \gamma}^{a b c}(k, p, q)=-i g f^{a b c}\left[\eta_{\alpha \beta}(p-k)_{\gamma}+\eta_{\alpha \gamma}(k-q)_{\beta}+\eta_{\beta \gamma}(q-p)_{\alpha}\right]=-i g f^{a b c} F_{\alpha \beta \gamma}(k, p, q),
$$

we have a new three-particle interaction vertex of one vector boson and two tensor gauge bosons - VTT. In momentum space it has the form

$$
\mathcal{V}_{\alpha \dot{\alpha} \beta \gamma \gamma \dot{\gamma}}^{a b c}(k, p, q)=-i g f^{a b c}\left[\eta_{\alpha \beta}(p-k)_{\gamma}+\eta_{\alpha \gamma}(k-q)_{\beta}+\eta_{\beta \gamma}(q-p)_{\alpha}\right] \eta_{\dot{\alpha} \dot{\gamma}}
$$

Notice that two parts in (35), which came from different terms of the Lagrangian $\mathcal{L}_{2}$, combine into the VVV vertex and the tensor $\eta_{\dot{\alpha} \dot{\gamma}}$. It is convenient to represent the vertex in the form

$$
\mathcal{V}_{\alpha \dot{\alpha} \beta \gamma \dot{\gamma}}^{a b c}(k, p, q)=-i g f^{a b c} F_{\alpha \dot{\alpha} \beta \gamma \dot{\gamma}}(k, p, q) .
$$

We have also a three-particle interaction vertex VTT of one vector boson and two tensor gauge bosons in the second Lagrangian $\mathcal{L}_{2}^{\prime}$. Explicitly the three-linear terms of Lagrangian $\mathcal{L}_{2}^{\prime}$ have the form: 


$$
\begin{aligned}
\mathcal{L}_{2}^{\prime} \text { cubic } & =\frac{1}{2} g f^{a b c}\left(\partial_{\mu} A_{\nu \lambda}^{a}-\partial_{\nu} A_{\mu \lambda}^{a}\right)\left(A_{\mu}^{b} A_{\lambda \nu}^{c}+A_{\mu \nu}^{b} A_{\lambda}^{c}\right) \\
& +\frac{1}{2} g f^{a b c}\left(\partial_{\mu} A_{\nu \nu}^{a}-\partial_{\nu} A_{\mu \nu}^{a}\right)\left(A_{\mu}^{b} A_{\lambda \lambda}^{c}+A_{\mu \lambda}^{b} A_{\lambda}^{c}\right) \\
& +\frac{1}{2} g f^{a b c}\left(\partial_{\mu} A_{\nu}^{a}-\partial_{\nu} A_{\mu}^{a}\right)\left(A_{\mu \nu}^{b} A_{\lambda \lambda}^{c}+A_{\mu \lambda}^{b} A_{\lambda \nu}^{c}\right)
\end{aligned}
$$

so that in momentum space we have

$$
\begin{aligned}
\mathcal{V}_{\alpha \alpha \dot{\alpha} \beta \gamma \dot{\gamma}}^{\prime a b c}(k, p, q) & =\frac{1}{2} i g f^{a b c} F_{\alpha \dot{\alpha} \beta \gamma \dot{\gamma}}^{\prime}(k, p, q) \\
F_{\alpha \dot{\alpha} \beta \gamma \dot{\gamma}}^{\prime}(k, p, q) & =(p-k)_{\gamma}\left(\eta_{\alpha \dot{\gamma}} \eta_{\dot{\alpha} \beta}+\eta_{\alpha \dot{\alpha}} \eta_{\beta \dot{\gamma}}\right) \\
& +(k-q)_{\beta}\left(\eta_{\alpha \dot{\gamma}} \eta_{\dot{\alpha} \gamma}+\eta_{\alpha \dot{\alpha}} \eta_{\gamma \dot{\gamma}}\right) \\
& +(q-p)_{\alpha}\left(\eta_{\dot{\alpha} \gamma} \eta_{\beta \dot{\gamma}}+\eta_{\dot{\alpha} \beta} \eta_{\gamma \dot{\gamma}}\right) \\
& +(p-k)_{\dot{\alpha}} \eta_{\alpha \beta} \eta_{\gamma \dot{\gamma}}+(p-k)_{\dot{\gamma}} \eta_{\alpha \beta} \eta_{\dot{\alpha} \gamma} \\
& +(k-q)_{\dot{\alpha}} \eta_{\alpha \gamma} \eta_{\beta \dot{\gamma}}+(k-q)_{\dot{\gamma}} \eta_{\alpha \gamma} \eta_{\dot{\alpha} \beta} \\
& +(q-p)_{\dot{\alpha}} \eta_{\beta \gamma} \eta_{\alpha \dot{\gamma}}+(q-p)_{\dot{\gamma}} \eta_{\alpha \dot{\alpha}} \eta_{\beta \gamma} .
\end{aligned}
$$

Collecting two terms of the three-point vertex VTT together we shall get

$$
\mathcal{V}_{\alpha \alpha \dot{\alpha} \beta \gamma \dot{\gamma}}^{t o t a b}(k, p, q)=\mathcal{V}_{\alpha \alpha \dot{\alpha} \beta \gamma \dot{\gamma}}^{a b c}(k, p, q)+\mathcal{V}_{\alpha \alpha \dot{\alpha} \beta \gamma \dot{\gamma}}^{\prime a b c}(k, p, q)
$$

Let us consider now four-particle interaction terms of the Lagrangian $\mathcal{L}_{2}+\mathcal{L}_{2}^{\prime}$. We have the standard four vector boson interaction vertex VVVV

$$
\begin{aligned}
\mathcal{V}_{\alpha \beta \gamma \delta}^{a b c d}(k, p, q, r)= & -g^{2} f^{l a c} f^{l b d}\left(\eta_{\alpha \beta} \eta_{\gamma \delta}-\eta_{\alpha \delta} \eta_{\beta \gamma}\right) \\
& -g^{2} f^{l a d} f^{l b c}\left(\eta_{\alpha \beta} \eta_{\gamma \delta}-\eta_{\alpha \gamma} \eta_{\beta \delta}\right) \\
& -g^{2} f^{l a b} f^{l c d}\left(\eta_{\alpha \gamma} \eta_{\beta \delta}-\eta_{\alpha \delta} \eta_{\beta \gamma}\right)
\end{aligned}
$$

and a new interaction of two vector and two tensor gauge bosons - the VVTT vertex,

$$
\begin{aligned}
\mathcal{L}_{2}^{\text {quartic }}= & -\frac{1}{4} g^{2} f^{a b c} f^{a b^{\prime}}\left(A_{\mu}^{b} A_{\nu \lambda}^{c}+A_{\mu \lambda}^{b} A_{\nu}^{c}\right)\left(A_{\mu}^{\dot{b}} A_{\nu \lambda}^{\dot{c}}+A_{\mu \lambda}^{\dot{b}} A_{\nu}^{c^{c}}\right) \\
& -\frac{1}{2} g^{2} f^{a b c} f^{a b^{\prime}} A_{\mu}^{b} A_{\nu}^{c} A_{\mu \lambda}^{\dot{b}} A_{\nu \lambda}^{\dot{c}},
\end{aligned}
$$

which in the momentum space will take the form

$$
\begin{aligned}
\mathcal{V}_{\alpha \beta \gamma \dot{\gamma} \delta \dot{\delta}}^{a b c d}(k, p, q, r)= & -g^{2} f^{l a c} f^{l b d}\left(\eta_{\alpha \beta} \eta_{\gamma \delta}-\eta_{\alpha \delta} \eta_{\beta \gamma}\right) \eta_{\dot{\gamma} \delta} \\
& -g^{2} f^{l a d} f^{l b c}\left(\eta_{\alpha \beta} \eta_{\gamma \delta}-\eta_{\alpha \gamma} \eta_{\beta \delta}\right) \eta_{\dot{\gamma} \delta} \\
& -g^{2} f^{l a b} f^{l c d}\left(\eta_{\alpha \gamma} \eta_{\beta \delta}-\eta_{\alpha \delta} \eta_{\beta \gamma}\right) \eta_{\dot{\gamma} \delta}
\end{aligned}
$$

The second part of the vertex VVTT comes from the Lagrangian $\mathcal{L}_{2}^{\prime}$ :

$$
\begin{aligned}
\mathcal{L}_{2}^{\prime \text { quartic }}= & +\frac{1}{4} g^{2} f^{a b c} f^{a \dot{b} \dot{c}}\left(A_{\mu}^{b} A_{\nu \lambda}^{c}+A_{\mu \lambda}^{b} A_{\nu}^{c}\right)\left(A_{\mu}^{\dot{b}} A_{\lambda \nu}^{\dot{c}}+A_{\mu \nu}^{\dot{b}} A_{\lambda}^{\dot{c}}\right) \\
& +\frac{1}{4} g^{2} f^{a b c} f^{a \dot{b} \dot{c}}\left(A_{\mu}^{b} A_{\nu \nu}^{c}+A_{\mu \nu}^{b} A_{\nu}^{c}\right)\left(A_{\mu}^{\dot{b}} A_{\lambda \lambda}^{\dot{c}}+A_{\mu \lambda}^{\dot{b}} A_{\lambda}^{\dot{c}}\right) \\
& +\frac{1}{2} g^{2} f^{a b c} f^{a b^{\prime}} A_{\mu}^{b} A_{\nu}^{c}\left(A_{\mu \nu}^{\dot{b}} A_{\lambda \lambda}^{\dot{c}}+A_{\mu \lambda}^{\dot{b}} A_{\lambda \nu}^{\dot{c}}\right),
\end{aligned}
$$


which in the momentum representation will take the form

$$
\begin{aligned}
2 \mathcal{V}_{\alpha \beta \gamma \gamma \dot{\gamma} \delta \dot{\delta}}^{\prime a b c d}(k, p, q, r)=g^{2} f^{l a c} f^{l b d}[ & +\eta_{\alpha \beta}\left(\eta_{\gamma \dot{\delta}} \eta_{\dot{\gamma} \delta}+\eta_{\gamma \dot{\gamma}} \eta_{\delta \dot{\delta}}\right) \\
& -\eta_{\beta \gamma}\left(\eta_{\alpha \dot{\delta}} \eta_{\dot{\gamma} \delta}+\eta_{\alpha \dot{\gamma}} \eta_{\delta \dot{\delta}}\right) \\
& -\eta_{\alpha \delta}\left(\eta_{\beta \dot{\gamma}} \eta_{\gamma \dot{\delta}}+\eta_{\beta \dot{\delta}} \eta_{\gamma \dot{\gamma}}\right) \\
& \left.+\eta_{\gamma \delta}\left(\eta_{\alpha \dot{\delta}} \eta_{\beta \dot{\gamma}}+\eta_{\alpha \dot{\gamma}} \eta_{\beta \dot{\delta}}\right)\right] \\
g^{2} f^{l a d} f^{l b c}[ & +\eta_{\alpha \beta}\left(\eta_{\gamma \dot{\delta}} \eta_{\dot{\gamma} \delta}+\eta_{\gamma \dot{\gamma}} \eta_{\delta \dot{\delta}}\right) \\
& -\eta_{\alpha \gamma}\left(\eta_{\beta \dot{\delta}} \eta_{\dot{\gamma} \delta}+\eta_{\beta \dot{\gamma}} \eta_{\delta \dot{\delta}}\right) \\
& -\eta_{\beta \delta}\left(\eta_{\alpha \dot{\gamma}} \eta_{\gamma \dot{\delta}}+\eta_{\alpha \dot{\delta}} \eta_{\gamma \dot{\gamma}}\right) \\
& \left.+\eta_{\gamma \delta}\left(\eta_{\alpha \dot{\gamma}} \eta_{\beta \dot{\delta}}+\eta_{\alpha \dot{\delta}} \eta_{\beta \dot{\gamma}}\right)\right] \\
& +\eta_{\alpha \gamma}\left(\eta_{\beta \dot{\gamma}} \eta_{\delta \dot{\delta}}+\eta_{\beta \dot{\delta}} \eta_{\delta \dot{\gamma}}\right) \\
& -\eta_{\beta \gamma}\left(\eta_{\alpha \dot{\gamma}} \eta_{\delta \dot{\delta}}+\eta_{\alpha \dot{\delta}} \eta_{\delta \dot{\gamma}}\right) \\
& -\eta_{\alpha \delta}\left(\eta_{\beta \dot{\delta}} \eta_{\gamma \dot{\gamma}}+\eta_{\beta \dot{\gamma}} \eta_{\gamma \dot{\delta}}\right) \\
& \left.+\eta_{\beta \delta}\left(\eta_{\alpha \dot{\delta}} \eta_{\gamma \dot{\gamma}}+\eta_{\alpha \dot{\gamma}} \eta_{\gamma \dot{\delta}}\right)\right] .
\end{aligned}
$$

The total vertex is

$$
\mathcal{V}_{\alpha \beta \gamma \delta}^{\text {tot abcd }}(k, p, q, r)=\mathcal{V}_{\alpha \beta \gamma \delta}^{a b c d}(k, p, q, r)+\mathcal{V}_{\alpha \beta \gamma \delta}^{\prime a b c d}(k, p, q, r)
$$

\section{Third-Rank Tensor Gauge Fields}

The Lagrangian $\mathcal{L}_{1}+g_{2}\left(\mathcal{L}_{2}+\mathcal{L}_{2}^{\prime}\right)$ contains the third-rank gauge fields $A_{\mu \nu \lambda}^{a}$, but without the corresponding kinetic term. In order to make the fields $A_{\mu \nu \lambda}^{a}$ dynamical we have added the corresponding Lagrangian $\mathcal{L}_{3}$ presented at the second line of the formula (12). But again the Lagrangian $\mathcal{L}_{3}$ is not the most general invariant which can be constructed from the corresponding field strength tensors. There are seven Lorentz invariant quadratic forms which form the second invariant Lagrangian $\mathcal{L}_{3}^{\prime}$ so that at this level the total Lagrangian is a sum

$$
\mathcal{L}=\mathcal{L}_{1}+g_{2}\left(\mathcal{L}_{2}+\mathcal{L}_{2}^{\prime}\right)+g_{3} \mathcal{L}_{3}+g_{3}^{\prime} \mathcal{L}_{3}^{\prime}+\ldots
$$

Indeed, the Lagrangian $\mathcal{L}_{3}$ has the form (12):

$$
\mathcal{L}_{3}=-\frac{1}{4} G_{\mu \nu, \lambda \rho}^{a} G_{\mu \nu, \lambda \rho}^{a}-\frac{1}{8} G_{\mu \nu, \lambda \lambda}^{a} G_{\mu \nu, \rho \rho}^{a}-\frac{1}{2} G_{\mu \nu, \lambda}^{a} G_{\mu \nu, \lambda \rho \rho}^{a}-\frac{1}{8} G_{\mu \nu}^{a} G_{\mu \nu, \lambda \lambda \rho \rho}^{a},
$$

where the field strength tensors (7) are

$$
\begin{aligned}
G_{\mu \nu, \lambda \rho \sigma}^{a}=\partial_{\mu} A_{\nu \lambda \rho \sigma}^{a}-\partial_{\nu} A_{\mu \lambda \rho \sigma}^{a}+g f^{a b c}\left\{A_{\mu}^{b} A_{\nu \lambda \rho \sigma}^{c}+A_{\mu \lambda}^{b} A_{\nu \rho \sigma}^{c}+A_{\mu \rho}^{b} A_{\nu \lambda \sigma}^{c}+A_{\mu \sigma}^{b} A_{\nu \lambda \rho}^{c}+\right. \\
\left.+A_{\mu \lambda \rho}^{b} A_{\nu \sigma}^{c}+A_{\mu \lambda \sigma}^{b} A_{\nu \rho}^{c}+A_{\mu \rho \sigma}^{b} A_{\nu \lambda}^{c}+A_{\mu \lambda \rho \sigma}^{b} A_{\nu}^{c}\right\}
\end{aligned}
$$

and

$$
\begin{aligned}
G_{\mu \nu, \lambda \rho \sigma \delta}^{a}=\partial_{\mu} A_{\nu \lambda \rho \sigma \delta}^{a}-\partial_{\nu} A_{\mu \lambda \rho \sigma \delta}^{a} & +g f^{a b c}\left\{A_{\mu}^{b} A_{\nu \lambda \rho \sigma \delta}^{c}+\sum_{\lambda \leftrightarrow \rho, \sigma, \delta} A_{\mu \lambda}^{b} A_{\nu \rho \sigma \delta}^{c}+\right. \\
& \left.+\sum_{\lambda, \rho \leftrightarrow \sigma, \delta} A_{\mu \lambda \rho}^{b} A_{\nu \sigma \delta}^{c}+\sum_{\lambda, \rho, \sigma \leftrightarrow \delta} A_{\mu \lambda \rho \sigma}^{b} A_{\nu \delta}^{c}+A_{\mu \lambda \rho \sigma \delta}^{b} A_{\nu}^{c}\right\} .
\end{aligned}
$$


The terms in parenthesis are symmetric over $\lambda \rho \sigma$ and $\lambda \rho \sigma \delta$ respectively. The Lagrangian $\mathcal{L}_{3}$ is invariant with respect to the extended gauge transformations (1) of the low-rank gauge fields $A_{\mu}, A_{\mu \nu}, A_{\mu \nu \lambda}$ and of the fourth-rank gauge field (3)

$$
\begin{array}{r}
\delta_{\xi} A_{\mu \nu \lambda \rho}=\partial_{\mu} \xi_{\nu \lambda \rho}-i g\left[A_{\mu}, \xi_{\nu \lambda \rho}\right]-i g\left[A_{\mu \nu}, \xi_{\lambda \rho}\right]-i g\left[A_{\mu \lambda}, \xi_{\nu \rho}\right]-i g\left[A_{\mu \rho}, \xi_{\nu \lambda}\right]- \\
-i g\left[A_{\mu \nu \lambda}, \xi_{\rho}\right]-i g\left[A_{\mu \nu \rho}, \xi_{\lambda}\right]-i g\left[A_{\mu \lambda \rho}, \xi_{\nu}\right]-i g\left[A_{\mu \nu \lambda \rho}, \xi\right]
\end{array}
$$

and also of the fifth-rank tensor gauge field (3)

$$
\begin{aligned}
\delta_{\xi} A_{\mu \nu \lambda \rho \sigma}= & \partial_{\mu} \xi_{\nu \lambda \rho \sigma}-i g\left[A_{\mu}, \xi_{\nu \lambda \rho \sigma}\right]-i g \sum_{\nu \leftrightarrow \lambda \rho \sigma}\left[A_{\mu \nu}, \xi_{\lambda \rho \sigma}\right]- \\
& -i g \sum_{\nu \lambda \leftrightarrow \rho \sigma}\left[A_{\mu \nu \lambda}, \xi_{\rho \sigma}\right]-i g \sum_{\nu \lambda \rho \leftrightarrow \sigma}\left[A_{\mu \nu \lambda \rho}, \xi_{\sigma}\right]-i g\left[A_{\mu \nu \lambda \rho}, \xi\right],
\end{aligned}
$$

where the gauge parameters $\xi_{\nu \lambda \rho}$ and $\xi_{\nu \lambda \rho \sigma}$ are totally symmetric rank-3 and rank-4 tensors. The extended gauge transformation of the higher-rank tensor gauge fields induces the gauge transformation of the fields strengths of the form (9)

$$
\begin{aligned}
\delta G_{\mu \nu, \lambda \rho \sigma}^{a}=g f^{a b c}\left(G_{\mu \nu, \lambda \rho \sigma}^{b} \xi^{c}+G_{\mu \nu, \lambda \rho}^{b}\right. & \xi_{\sigma}^{c}+G_{\mu \nu, \lambda \sigma}^{b} \xi_{\rho}^{c}+G_{\mu \nu, \rho \sigma}^{b} \xi_{\lambda}^{c}+ \\
& \left.+G_{\mu \nu, \lambda}^{b} \xi_{\rho \sigma}^{c}+G_{\mu \nu, \rho}^{b} \xi_{\lambda \sigma}^{c}+G_{\mu \nu, \sigma}^{b} \xi_{\lambda \rho}^{c}+G_{\mu \nu}^{b} \xi_{\lambda \rho \sigma}^{c}\right)
\end{aligned}
$$

and

$$
\begin{aligned}
\delta G_{\mu \nu, \lambda \rho \sigma \delta}^{a}=g f^{a b c}\left(G_{\mu \nu, \lambda \rho \sigma \delta}^{b} \xi^{c}\right. & +\sum_{\lambda \rho, \sigma \leftrightarrow \delta} G_{\mu \nu, \lambda \rho \sigma}^{b} \xi_{\delta}^{c}+ \\
& \left.+\sum_{\lambda \rho \leftrightarrow \sigma, \delta} G_{\mu \nu, \lambda \rho}^{b} \xi_{\sigma \delta}^{c}+\sum_{\lambda \leftrightarrow \rho, \sigma, \delta} G_{\mu \nu, \lambda}^{b} \xi_{\rho \sigma \delta}^{c}+G_{\mu \nu}^{b} \xi_{\lambda \rho \sigma \delta}^{c}\right) .
\end{aligned}
$$

Using the above homogeneous transformations for the field strength tensors one can demonstrate the invariance of the Lagrangian $\mathcal{L}_{3}$ with respect to the extended gauge transformations (8), (49) and (49) (see reference [23] for details).

Our purpose now is to present a second invariant Lagrangian which can be constructed in terms of the above field strength tensors. Let us consider the following seven Lorentz invariant quadratic forms which can be constructed by the corresponding field strength tensors

$$
\begin{gathered}
G_{\mu \nu, \lambda \rho}^{a} G_{\mu \lambda, \nu \rho}^{a}, \quad G_{\mu \nu, \nu \lambda}^{a} G_{\mu \rho, \rho \lambda}^{a}, \quad G_{\mu \nu, \nu \lambda}^{a} G_{\mu \lambda, \rho \rho}^{a}, \quad G_{\mu \nu, \lambda}^{a} G_{\mu \lambda, \nu \rho \rho}^{a}, \\
G_{\mu \nu, \lambda}^{a} G_{\mu \rho, \nu \lambda \rho}^{a}, \quad G_{\mu \nu, \nu}^{a} G_{\mu \lambda, \lambda \rho \rho}^{a}, \quad G_{\mu \nu}^{a} G_{\mu \lambda, \nu \lambda \rho \rho}^{a} .
\end{gathered}
$$

Calculating the variation of each of these terms with respect to the gauge transformation (8), (49) and (49) one can get convinced that the particular linear combination

$$
\begin{aligned}
\mathcal{L}_{3}^{\prime} & =\frac{1}{4} G_{\mu \nu, \lambda \rho}^{a} G_{\mu \lambda, \nu \rho}^{a}+\frac{1}{4} G_{\mu \nu, \nu \lambda}^{a} G_{\mu \rho, \rho \lambda}^{a}+\frac{1}{4} G_{\mu \nu, \nu \lambda}^{a} G_{\mu \lambda, \rho \rho}^{a} \\
& +\frac{1}{4} G_{\mu \nu, \lambda}^{a} G_{\mu \lambda, \nu \rho \rho}^{a}+\frac{1}{2} G_{\mu \nu, \lambda}^{a} G_{\mu \rho, \nu \lambda \rho}^{a}+\frac{1}{4} G_{\mu \nu, \nu}^{a} G_{\mu \lambda, \lambda \rho \rho}^{a}+\frac{1}{4} G_{\mu \nu}^{a} G_{\mu \lambda, \nu \lambda \rho \rho}^{a}
\end{aligned}
$$

forms an invariant Lagrangian. Indeed, the variation of the first term is

$$
\delta_{\xi} G_{\mu \nu, \lambda \rho}^{a} G_{\mu \lambda, \nu \rho}^{a}=2 g f^{a b c} G_{\mu \nu, \lambda \rho}^{a} G_{\mu \lambda, \nu}^{b} \xi_{\rho}^{c}+2 g f^{a b c} G_{\mu \nu, \lambda \rho}^{a} G_{\mu \lambda, \rho}^{b} \xi_{\nu}^{c}+2 g f^{a b c} G_{\mu \nu, \lambda \rho}^{a} G_{\mu \lambda}^{b} \xi_{\nu \rho}^{c},
$$


of the second term is

$$
\delta_{\xi} G_{\mu \nu, \nu \lambda}^{a} G_{\mu \rho, \rho \lambda}^{a}=2 g f^{a b c} G_{\mu \nu, \nu \lambda}^{a} G_{\mu \rho, \rho}^{b} \xi_{\lambda}^{c}+2 g f^{a b c} G_{\mu \nu, \nu \lambda}^{a} G_{\mu \rho, \lambda}^{b} \xi_{\rho}^{c}+2 g f^{a b c} G_{\mu \nu, \nu \lambda}^{a} G_{\mu \rho}^{b} \xi_{\rho \lambda}^{c},
$$

of the third term is

$$
\begin{aligned}
\delta_{\xi} G_{\mu \nu, \nu \lambda}^{a} G_{\mu \lambda, \rho \rho}^{a}=2 g f^{a b c} G_{\mu \nu, \nu \lambda}^{a} G_{\mu \lambda, \rho}^{b} \xi_{\rho}^{c}+ & g f^{a b c} G_{\mu \nu, \nu \lambda}^{a} G_{\mu \lambda}^{b} \xi_{\rho \rho}^{c}+g f^{a b c} G_{\mu \lambda, \rho \rho}^{a} G_{\mu \nu, \nu}^{b} \xi_{\lambda}^{c}+ \\
& +g f^{a b c} G_{\mu \lambda, \rho \rho}^{a} G_{\mu \nu, \lambda}^{b} \xi_{\nu}^{c}+g f^{a b c} G_{\mu \lambda, \rho \rho}^{a} G_{\mu \nu}^{b} \xi_{\nu \lambda}^{c}
\end{aligned}
$$

of the forth term is

$$
\begin{aligned}
\delta_{\xi} G_{\mu \nu, \lambda}^{a} G_{\mu \lambda, \nu \rho \rho}^{a}= & g f^{a b c} G_{\mu \lambda, \nu \rho \rho}^{a} G_{\mu \nu}^{b} \xi_{\lambda}^{c}+2 g f^{a b c} G_{\mu \lambda, \nu \rho}^{a} G_{\mu \nu, \lambda}^{b} \xi_{\rho}^{c}+g f^{a b c} G_{\mu \lambda, \rho \rho}^{a} G_{\mu \nu, \lambda}^{b} \xi_{\nu}^{c}+ \\
& +g f^{a b c} G_{\mu \nu, \lambda}^{a} G_{\mu \lambda, \nu}^{b} \xi_{\rho \rho}^{c}+2 g f^{a b c} G_{\mu \nu, \lambda}^{a} G_{\mu \lambda, \rho}^{b} \xi_{\nu \rho}^{c}+g f^{a b c} G_{\mu \nu, \lambda}^{a} G_{\mu \lambda}^{b} \xi_{\nu \rho \rho}^{c},
\end{aligned}
$$

of the fifth term is

$$
\begin{array}{r}
\delta_{\xi} G_{\mu \nu, \lambda}^{a} G_{\mu \rho, \nu \lambda \rho}^{a}= \\
g f^{a b c} G_{\mu \rho, \nu \lambda \rho}^{a} G_{\mu \nu}^{b} \xi_{\lambda}^{c}+g f^{a b c} G_{\mu \rho, \nu \lambda}^{b} G_{\mu \nu, \lambda}^{a} \xi_{\rho}^{c}+g f^{a b c} G_{\mu \rho, \nu \rho}^{b} G_{\mu \nu, \lambda}^{a} \xi_{\lambda}^{c}+g f^{a b c} G_{\mu \rho, \lambda \rho}^{b} G_{\mu \nu, \lambda}^{a} \xi_{\nu}^{c}+ \\
+g f^{a b c} G_{\mu \nu, \lambda}^{a} G_{\mu \rho, \nu}^{b} \xi_{\lambda \rho}^{c}+g f^{a b c} G_{\mu \nu, \lambda}^{a} G_{\mu \rho, \lambda}^{b} \xi_{\nu \rho}^{c}+g f^{a b c} G_{\mu \nu, \lambda}^{a} G_{\mu \rho, \rho}^{b} \xi_{\nu \lambda}^{c}+g f^{a b c} G_{\mu \nu, \lambda}^{a} G_{\mu \rho}^{b} \xi_{\nu \lambda \rho}^{c},
\end{array}
$$

of the sixth term is

$$
\begin{array}{r}
\delta_{\xi} G_{\mu \nu, \nu}^{a} G_{\mu \lambda, \lambda \rho \rho}^{a}= \\
g f^{a b c} G_{\mu \lambda, \lambda \rho \rho}^{a} G_{\mu \nu}^{b} \xi_{\nu}^{c}+2 g f^{a b c} G_{\mu \lambda, \lambda \rho}^{b} G_{\mu \nu, \nu}^{a} \xi_{\rho}^{c}+g f^{a b c} G_{\mu \lambda, \rho \rho}^{b} G_{\mu \nu, \nu}^{a} \xi_{\lambda}^{c}+g f^{a b c} G_{\mu \lambda, \lambda}^{b} G_{\mu \nu, \nu}^{a} \xi_{\rho \rho}^{c}+ \\
+2 g f^{a b c} G_{\mu \lambda, \rho}^{b} G_{\mu \nu, \nu}^{a} \xi_{\lambda \rho}^{c}+g f^{a b c} G_{\mu \nu, \nu}^{a} G_{\mu \lambda}^{b} \xi_{\lambda \rho \rho}^{c}
\end{array}
$$

and finally of the seventh term is

$$
\begin{array}{r}
\delta_{\xi} G_{\mu, \nu}^{a} G_{\mu \lambda, \nu \lambda \rho \rho}^{a}= \\
2 g f^{a b c} G_{\mu \nu}^{a} G_{\mu \lambda, \nu \lambda \rho}^{b} \xi_{\rho}^{c}+g f^{a b c} G_{\mu \nu}^{a} G_{\mu \lambda, \nu \rho \rho}^{b} \xi_{\lambda}^{c}+g f^{a b c} G_{\mu \nu}^{a} G_{\mu \lambda, \lambda \rho \rho}^{b} \xi_{\nu}^{c}+g f^{a b c} G_{\mu \nu}^{a} G_{\mu \lambda, \nu \lambda}^{b} \xi_{\rho \rho}^{c}+ \\
2 g f^{a b c} G_{\mu \nu}^{a} G_{\mu \lambda, \nu \rho}^{b} \xi_{\lambda \rho}^{c}+2 g f^{a b c} G_{\mu \nu}^{a} G_{\mu \lambda, \lambda \rho}^{b} \xi_{\nu \rho}^{c}+g f^{a b c} G_{\mu \nu}^{a} G_{\mu \lambda, \rho \rho}^{b} \xi_{\nu \lambda}^{c}+ \\
g f^{a b c} G_{\mu \nu}^{a} G_{\mu \lambda, \nu}^{b} \xi_{\lambda \rho \rho}^{c}+g f^{a b c} G_{\mu \nu}^{a} G_{\mu \lambda, \lambda}^{b} \xi_{\nu \rho \rho}^{c}+2 g f^{a b c} G_{\mu \nu}^{a} G_{\mu \lambda, \rho}^{b} \xi_{\nu \lambda \rho}^{c}+g f^{a b c} G_{\mu \nu}^{a} G_{\mu \lambda}^{b} \xi_{\nu \lambda \rho \rho}^{c} .
\end{array}
$$

Some of the terms here are equal to zero, like: $g f^{a b c} G_{\mu \nu, \lambda}^{a} G_{\mu \rho, \lambda}^{b} \xi_{\nu \rho}^{c}, g f^{a b c} G_{\mu \lambda, \lambda}^{a} G_{\mu \nu, \nu}^{b} \xi_{\rho \rho}^{c}$ and $g f^{a b c} G_{\mu \nu}^{a} G_{\mu \lambda}^{b} \xi_{\nu \lambda \rho \rho}^{c}$. Amazingly all nonzero terms cancel each other.

In general one should construct all invariant quadratic forms for all tensor gauge fields. A regular way of construction of all invariant Lagrangians at every level is not known to the author, with exception of the case of infinite series of particular invariants presented in [23] by the formulas (10) and (11).

\section{$5 \quad$ Extended Polygauge Groups}

We have three possible extensions of the Yang-Mills gauge group. The first extension was defined by the equations (1) and (2). It allows definition of field strength tensors (6) and (7) transforming homogeneously with respect to the extended gauge transformation (8) and (9). 
The second extension was also defined in [23] for the higher-rank tensor fields $A_{\lambda_{1} \ldots \lambda_{s}}$ which are totally symmetric tensors. In that case the gauge transformation (1), (2) should be modified in order to respect the symmetry properties of the tensor fields. Therefore the extended gauge transformation (1), (2) was symmetrized over all indices as follows:

$$
\begin{aligned}
\tilde{\delta} A_{\mu}^{a} & =\left(\delta^{a b} \partial_{\mu}+g f^{a c b} A_{\mu}^{c}\right) \xi^{b}, \\
\tilde{\delta} A_{\mu \nu}^{a} & =\underline{\left(\delta^{a b} \partial_{\mu}+g f^{a c b} A_{\mu}^{c}\right) \xi_{\nu}^{b}}+g f^{a c b} A_{\mu \nu}^{c} \xi^{b}, \\
\tilde{\delta} A_{\mu \nu \lambda}^{a} & =\underline{\left(\delta^{a b} \partial_{\mu}+g f^{a c b} A_{\mu}^{c}\right) \xi_{\nu \lambda}^{b}}+g f^{a c b}\left(\underline{A_{\mu \nu}^{c} \xi_{\lambda}^{b}}+A_{\mu \nu \lambda}^{c} \xi^{b}\right),
\end{aligned}
$$

where

$$
\begin{aligned}
\frac{\left(\delta^{a b} \partial_{\mu}+g f^{a c b} A_{\mu}^{c}\right) \xi_{\nu}^{b}}{\underline{A_{\mu \nu}^{c} \xi_{\lambda}^{b}}} & =\left(\delta^{a b} \partial_{\mu}+g f^{a c b} A_{\mu}^{c}\right) \xi_{\nu}^{b}+\left(\delta^{a b} \partial_{\nu}+g f^{a c b} A_{\mu}^{c}\right) \xi_{\mu}^{b} \\
& A_{\nu \lambda}^{c} \xi_{\mu}^{b}+A_{\lambda \mu}^{c} \xi_{\nu}^{b},
\end{aligned}
$$

and so on. One should also require that the gauge parameters $\xi_{\lambda_{1} \ldots \lambda_{s}}^{a}$ are totally symmetric tensors. For example, in a matrix notation, we have

$$
\tilde{\delta}_{\xi} A_{\mu \nu}=\partial_{\mu} \xi_{\nu}-i g\left[A_{\mu}, \xi_{\nu}\right]+\partial_{\nu} \xi_{\mu}-i g\left[A_{\nu}, \xi_{\mu}\right]-i g\left[A_{\mu \nu}, \xi\right] .
$$

The commutator of the above gauge transformations acting on a rank-s tensor is again a gauge transformation

$$
\left[\tilde{\delta}_{\eta}, \tilde{\delta}_{\xi}\right] A_{\lambda_{1} \ldots \lambda_{s}}=-i g \tilde{\delta}_{\zeta} A_{\lambda_{1} \ldots \lambda_{s}}
$$

and the gauge parameters form the same algebra as before (5). Therefore we concluded that the symmetrized gauge transformations also form a closed algebraic structure [23].

The third extension of the gauge transformations with double number of gauge parameters can be defined as follows:

$$
\begin{aligned}
\delta_{\xi} A_{\mu}^{a} & =\left(\delta^{a b} \partial_{\mu}+g f^{a c b} A_{\mu}^{c}\right) \xi^{b}, \\
\delta_{\xi} A_{\mu \nu}^{a} & =\left(\delta^{a b} \partial_{\mu}+g f^{a c b} A_{\mu}^{c}\right) \xi_{\nu}^{b}+g f^{a c b} A_{\mu \nu}^{c} \xi^{b}, \\
\delta_{\eta} A_{\mu \nu}^{a} & =\left(\delta^{a b} \partial_{\nu}+g f^{a c b} A_{\nu}^{c}\right) \eta_{\mu}^{b}+g f^{a c b} A_{\mu \nu}^{c} \eta^{b},
\end{aligned}
$$

and also forms a closed algebraic structure.

\section{Conclusion}

The transformations considered in the previous sections enlarge the original algebra of Abelian local gauge transformations found in [61] (expression (64) in [61]) to a nonAbelian case and unify into one multiplet particles with arbitrary spins and with linearly growing multiplicity. As we have seen, this leads to a natural generalization of the YangMills theory. The extended non-Abelian gauge transformations defined for the tensor gauge fields led to the construction of the appropriate field strength tensors and of the invariant Lagrangians. The proposed extension may lead to a natural inclusion of the standard theory of fundamental forces into a larger theory in which standard particles 
(vector gauge bosons, leptons and quarks) represent a low-spin subgroup of an enlarged family of particles with higher spins.

As an example of an extended gauge field theory with infinite many gauge fields, this theory can be associated with the field theory of the tensionless strings, because in our generalization of the non-Abelian Yang-Mills theory we essentially used the symmetry group which appears as symmetry of the ground state wave function of the tensionless strings $[61,60,62]$. Nevertheless I do not know how to derive it directly from tensionless strings, therefore one can not claim that they are indeed identical. The main reason is that the above construction, which is purely field-theoretical, has a great advantage of being well defined on and off the mass-shell, while the string-theoretical constructions have not been yet developed to the same level, because the corresponding vertex operators are well defined only on the mass-shell [62]. The tensor gauge field theory could probably be a genuine tensionless string field theory because of the common symmetry group, and

it would be useful to understand, whether the string theory can fully reproduce this result. Discussion of the tensionless strings and related questions can also be found in $[63,64,65,66,67,68,69,70,71,72,73,74,75,76,77,78,79,80]$.

This work was partially supported by the EEC Grant no. HPRN-CT-1999-00161 and EEC Grant no. MRTN-CT-2004-005616.

\section{References}

[1] C.N.Yang and R.L.Mills. "Conservation of Isotopic Spin and Isotopic Gauge Invariance". Phys. Rev. 96 (1954) 191

[2] H.Yukawa. "On the Interaction of Elementary Particles" I, Proc. Phys. Math. Soc. Japan 17 (1935) 48

[3] J. S. Schwinger, "A Theory Of The Fundamental Interactions," Annals Phys. 2 (1957) 407.

[4] S. L. Glashow, "Partial Symmetries Of Weak Interactions," Nucl. Phys. 22 (1961) 579 .

[5] A.Salam, In Elementary Particle Theory: Relativistic Groups and Analyticity (Nobel Symposium No.8), edited by N.Svartholm, (Almqvist and Wiksell, Stockholm,1968)

[6] S.Weinberg. A model of Leptons. Phys. Rev. Lett. 19 (1967) 1264

[7] R. P. Feynman, "Quantum Theory Of Gravitation," Acta Phys. Polon. 24 (1963) 697.

[8] L. D. Faddeev and V. N. Popov, "Feynman Diagrams For The Yang-Mills Field," Phys. Lett. B 25 (1967) 29; "Perturbation Theory For Gauge Invariant Fields," FERMILAB-PUB-72-057-T

[9] B. S. DeWitt, "Quantum Theory Of Gravity. II,III," Phys. Rev. 162 (1967) 1195,1239 .

[10] S. Mandelstam, "Feynman Rules For Electromagnetic And Yang-Mills Fields From The Gauge Independent Field Theoretic Formalism," Phys. Rev. 175 (1968) 1580. 
[11] A. A. Slavnov and L. D. Faddeev, "Massless And Massive Yang-Mills Field. (In Russian)," Theor. Math. Phys. 3 (1970) 312 [Teor. Mat. Fiz. 3 (1970) 18].

[12] G. 't Hooft, "Renormalization Of Massless Yang-Mills Fields," Nucl. Phys. B 33 (1971) 173.

[13] G. 't Hooft, "Renormalizable Lagrangians For Massive Yang-Mills Fields," Nucl. Phys. B 35 (1971) 167.

[14] G. 't Hooft and M. J. G. Veltman, "Regularization And Renormalization Of Gauge Fields," Nucl. Phys. B 44 (1972) 189.

[15] B. W. Lee, "Renormalizable Massive Vector Meson Theory. Perturbation Theory Of The Higgs Phenomenon," Phys. Rev. D 5 (1972) 823.

[16] B. W. Lee and J. Zinn-Justin, "Spontaneously Broken Gauge Symmetries. I,II,III." Phys. Rev. D 5 (1972) 3121,3137,3155.

[17] A. A. Slavnov, "Ward Identities In Gauge Theories," Theor. Math. Phys. 10 (1972) 99 [Teor. Mat. Fiz. 10 (1972) 153].

[18] J. C. Taylor, "Ward Identities And Charge Renormalization Of The Yang-Mills Field," Nucl. Phys. B 33 (1971) 436.

[19] D. J. Gross and F. Wilczek, "Ultraviolet Behavior Of Non-Abelian Gauge Theories," Phys. Rev. Lett. 30, 1343 (1973).

[20] H. D. Politzer, "Reliable Perturbative Results For Strong Interactions?," Phys. Rev. Lett. 30, 1346 (1973).

[21] H. Fritzsch, M. Gell-Mann and H. Leutwyler, "Advantages Of The Color Octet Gluon Picture," Phys. Lett. B 47 (1973) 365.

[22] G. K. Savvidy, "Infrared Instability Of The Vacuum State Of Gauge Theories And Asymptotic Freedom," Phys. Lett. B 71 (1977) 133.

[23] G. Savvidy, "Generalization of Yang-Mills theory: Non-Abelian tensor gauge fields and higher-spin extension of standard model," arXiv:hep-th/0505033.

[24] V.Fock. Über die invariante Form der Wellen- und der Bewegungsgleichungen für einen geladenen Massenpunkt. Z. für Physik 39 (1927) 226

[25] O.Klein. Quantentheorie und fünfdimensionale Relativitätstheorie. Z. für Physik 37 (1926) 895

[26] F.London. Quantenmechanische Deutung der Theorie von Weyl. Z. für Physik 42 (1927) 375

[27] H.Weyl. "Elektron und Gravitation" Z. für Physik 56 (1929) 330;

H.Weyl. Gruppentheorie und Quantenmechanik. Ch.IV. Leipzig,1928 
[28] W.Pauli und W.Heisenberg. " Zur Quantentheorie der Wellenfelder. II" Z. für Physik 59 (1930) 168;

W.Pauli. Relativistic Field Theories of Elementary Particles. Rev. Mod. Phys. 13 (1941) 203

[29] S.S.Chern. Topics in Defferential Geometry, Ch. III "Theory of Connections" (The Institute for Advanced Study, Princeton, 1951)

[30] E.Majorana. Teoria Relativistica di Particelle con Momento Intrinseco Arbitrario. Nuovo Cimento 9 (1932) 335

[31] P.A.M.Dirac. Relativistic wave equations. Proc. Roy. Soc. A155 (1936) 447; Unitary Representation of the Lorentz Group. Proc. Roy. Soc. A183 (1944) 284.

[32] M. Fierz. Helv. Phys. Acta. 12 (1939) 3.

M. Fierz and W. Pauli. On Relativistic Wave Equations for Particles of Arbitrary Spin in an Electromagnetic Field. Proc. Roy. Soc. A173 (1939) 211.

[33] E. Wigner. On Unitary Representations of the Inhomogeneous Lorentz Group. Ann. Math. 40 (1939) 149.

[34] W. Rarita and J. Schwinger. On a Theory of Particles with Half-Integral Spin. Phys. Rev. 60 (1941) 61

[35] H.Yukawa. "Quantum Theory of Non-Local Fields. Part I. Free Fields" Phys. Rev. 77 (1950) 219

M. Fierz. "Non-Local Fields" Phys. Rev. 78 (1950) 184

[36] J.Schwinger. Particles, Sourses, and Fields (Addison-Wesley, Reading, MA, 1970)

[37] S. Weinberg, "Feynman Rules For Any Spin," Phys. Rev. 133 (1964) B1318.

[38] S. Weinberg, "Feynman Rules For Any Spin. 2: Massless Particles," Phys. Rev. 134 (1964) B882.

[39] S. Weinberg, "Photons And Gravitons In S Matrix Theory: Derivation Of Charge Conservation And Equality Of Gravitational And Inertial Mass," Phys. Rev. 135 (1964) B1049.

[40] S. J. Chang. Lagrange Formulation for Systems with Higher Spin. Phys.Rev. 161 (1967) 1308

[41] L. P. S. Singh and C. R. Hagen. Lagrangian formulation for arbitrary spin. I. The boson case. Phys. Rev. D9 (1974) 898

[42] L. P. S. Singh and C. R. Hagen. Lagrangian formulation for arbitrary spin. II. The fermion case. Phys. Rev. D9 (1974) 898, 910

[43] C.Fronsdal. Massless fields with integer spin, Phys.Rev. D18 (1978) 3624

[44] J.Fang and C.Fronsdal. Massless fields with half-integral spin, Phys. Rev. D18 (1978) 3630 
[45] N.S.Gupta. "Gravitation and Electromagnetism". Phys. Rev. 96, (1954) 1683.

[46] R.H.Kraichnan. "Special-relativistic derivation of generally covariant gravitation theory". Phys. Rev. 98, (1955) 1118.

[47] W.E.Thirring. "An alternative approach to the theory of gravitation". Ann. Phys. 16, (1961) 96.

[48] R.P.Feynman. "Feynman Lecture on Gravitation". Westview Press 2002.

[49] S.Deser. "Self-interaction and gauge invariance". Gen. Rel. Grav. 1, (1970) 9.

[50] J.Fang and C.Fronsdal. "Deformation of gauge groups. Gravitation". J. Math. Phys. 20 (1979) 2264

[51] S.Weinberg and E.Witten, "Limits on massless particles," Phys. Lett. B 96 (1980) 59.

[52] C. Aragone and S. Deser, "Constraints on gravitationally coupled tensor fields," Nuovo. Cimento. 3A (1971) 709;

C.Aragone and S.Deser, "Consistancy problems of hypergavity," Phys. Lett. B 86 (1979) 161.

[53] F. A. Berends, G. J. H Burgers and H. Van Dam, "On the Theoretical problems in Constructing Interactions Involving Higher-Spin Massless Particles," Nucl. Phys. B 260 (1985) 295.

[54] B.deWit, F.A. Berends, J.W.van Halten and P.Nieuwenhuizen, "On Spin-5/2 gauge fields," J. Phys. A: Math. Gen. 16 (1983) 543.

[55] M.A.Vasiliev, "Progress in Higher Spin Gauge Theories" hep-th/0104246; M.A.Vasiliev et. al. "Nonlinear Higher Spin Theories in Various Domensions" hepth/0503128

[56] E. Sezgin and P. Sundell, "Holography in 4D (Super) Higher Spin Theories and a Test via Cubic Scalar Couplings," hep-th/0305040

[57] A. Sagnotti, E. Sezgin and P. Sundell, "On higher spins with a strong $\operatorname{Sp}(2, \mathrm{R})$ condition," arXiv:hep-th/0501156.

[58] A. K. Bengtsson, I. Bengtsson and L. Brink, "Cubic Interaction Terms For Arbitrary Spin," Nucl. Phys. B 227 (1983) 31.

[59] A. K. Bengtsson, I. Bengtsson and L. Brink, "Cubic Interaction Terms For Arbitrarily Extended Supermultiplets," Nucl. Phys. B 227 (1983) 41.

[60] G. K. Savvidy, Conformal Invariant Tensionless Strings, Phys. Lett. B 552 (2003) 72 .

[61] G. K. Savvidy, "Tensionless strings: Physical Fock space and higher spin fields," Int. J. Mod. Phys. A 19, (2004) 3171-3194. 
[62] G. Savvidy, "Tensionless strings, correspondence with SO(D,D) sigma model," Phys. Lett. B 615 (2005) 285.

[63] L. Edgren, R. Marnelius and P. Salomonson, "Infinite spin particles," JHEP 0505 (2005) 002 [arXiv:hep-th/0503136].

[64] N. Turok, M. Perry and P. J. Steinhardt, "M theory model of a big crunch / big bang transition," Phys. Rev. D 70 (2004) 106004 [arXiv:hep-th/0408083].

[65] J. Engquist and P. Sundell, "Brane partons and singleton strings," arXiv:hepth/0508124.

[66] J. Mourad, "Continuous spin particles from a string theory," arXiv:hep-th/0504118.

[67] X. Bekaert, S. Cnockaert, C. Iazeolla and M. A. Vasiliev, "Nonlinear higher spin theories in various dimensions," arXiv:hep-th/0503128.

[68] L. Brink, "Particle physics as representations of the Poincare algebra," arXiv:hepth/0503035.

[69] J. Gamboa, M. Loewe and F. Mendez, "Quantum theory of tensionless noncommutative p-branes," Phys. Rev. D 70 (2004) 106006.

[70] G. Bonelli, "On the boundary gauge dual of closed tensionless free strings in AdS," JHEP 0411 (2004) 059 [arXiv:hep-th/0407144].

[71] I. Bakas and C. Sourdis, "On the tensionless limit of gauged WZW models," JHEP 0406 (2004) 049 [arXiv:hep-th/0403165].

[72] A. Bredthauer, U. Lindstrom, J. Persson and L. Wulff, 'Type IIB tensionless superstrings in a pp-wave background," JHEP 0402 (2004) 051 [arXiv:hep-th/0401159].

[73] J. Gamboa and F. Mendez, "Statistical quantum mechanics of many universes," arXiv:hep-th/0304116.

[74] W. F. Chagas-Filho, "Symmetries in particle and string theories," arXiv:hepth/0309219.

[75] M. Bianchi, J. F. Morales and H. Samtleben, "On stringy AdS(5)x $S^{5}$ and higher spin holography," JHEP 0307 (2003) 062 [arXiv:hep-th/0305052].

[76] E. Gabrielli, "Extended Pure Yang-Mills Gauge Theories With Scalar And Tensor Gauge Phys. Lett. B 258 (1991) 151.

[77] E. Gabrielli, "Extended gauge theories in Euclidean space with higher spin fields," Annals Phys. 287 (2001) 229 [arXiv:hep-th/9909117].

[78] J. C. Baez, "Higher Yang-Mills theory," arXiv:hep-th/0206130.

[79] C. Castro, "Generalized p-forms electrodynamics in Clifford spaces," Mod. Phys. Lett. A 19 (2004) 19.

[80] A. K. H. Bengtsson, J. Math. Phys. 46 (2005) 042312 [arXiv:hep-th/0403267]. 